influenza virus, has been reported in ostriches in South Africa and Denmark. Chicks are apparently more susceptible than are adults, but all age groups can be affected. Clinically, infected birds show severe depression, respiratory signs, ocular discharge and green urates. Treatment is usually unsuccessful, and vaccination relies on the identification of the viral strain present. Prevention is best achieved by preventing contact between ostriches and free-ranging birds. ${ }^{35}$

\section{DISEASES OF THE NERVOUS SYSTEM}

Newcastle disease, caused by paramyxovirus serotype 1 (PMV1), has been reported in ostriches in Africa and Israel and in rheas in Brazil. The virus is documented in 236 avian species, confirming widespread susceptibility of birds to this disease ${ }^{58}$ Young birds appear to be more susceptible, with up to $50 \%$ mortality. Affected birds initially show a slight head tilt, frequent scratching of the head and a tic of the neck muscles. This progresses on to torticollis, uncontrolled head movements and finally an inability to lift the head off the ground. Death occurs in 2 to 3 days. ${ }^{35}$ There is no treatment, although some birds recover spontaneously. Vaccination is carried out on some farms in South Africa with apparently good results. $9,57,67$

Western equine encephalitis (WEE) has been reported in emus and rheas in North America. ${ }^{69}$ Affected birds are initially depressed, progressing on to paresis, recumbency and finally paralysis. Mortality rates are less than $20 \%$, with recumbent birds dying within 48 hours. Vector control and vaccination are usually effective in preventing this disease. ${ }^{58}$

Borna Disease is seen in 2- to 8-week-old ostrich chicks in Israel. Affected chicks show paresis and reluctance to move, progressing on to paralysis and finally death from dehydration..$^{58}$

Cerebral nematodiasis has been reported in ostriches and emus in North America. Chandlerella quiscali and Baylisascaris spp. have been isolated from birds showing signs including ataxia, abnormal gait, muscle weakness, recumbency and death. ${ }^{21}$

\section{DISEASES OF THE REPRODUCTIVE SYSTEM}

\section{Disorders of the Female Reproductive Tract}

The quality of the egg and its shell can be a reflection of the health of the hen and reproductive tract that produced it; consequently, veterinarians are occasionally called on to comment on abnormal eggs. A number of abnormalities have been observed (Table 41.14).
Table 41.14 | Assessment of Egg Quality Observed Abnormality Possible Causes

\begin{tabular}{|l|l|}
\hline Shell too thin & $\begin{array}{l}\text { - Immaturity of hen } \\
\text { - Malnutrition } \\
\text { - Salpingitis causing rapid transit of egg } \\
\text { through tract } \\
\text { - Genetics }\end{array}$ \\
\hline Shell too thick & - Excessive calcium supplementation \\
\hline $\begin{array}{l}\text { "Stress lines" - } \\
\text { ridging and grooving } \\
\text { of the egg shell }\end{array}$ & $\begin{array}{l}\text { - Salpingitis due to infection or excessive } \\
\text { egg laying }\end{array}$ \\
\hline Shell texture chalky & $\begin{array}{l}\text { - Impopper deficiency } \\
\text { - First eggs of the season }\end{array}$ \\
\hline Blood on the shell & $\begin{array}{l}\text { - Maiden hen } \\
\text { - Salpingitis }\end{array}$ \\
\hline Excessive porosity & $\begin{array}{l}\text { - Immaturity of hen } \\
\text { - Start or end of laying season } \\
\text { - Nutrition } \\
\text { - Genetics }\end{array}$ \\
\hline Floating air cell & $\begin{array}{l}\text { - Rough handling by bird or people } \\
\text { - Genetics }\end{array}$ \\
\hline Cracked or damaged shells & $\begin{array}{l}\text { - Eggs laid while standing up } \\
\text { - Rough handling by bird or people }\end{array}$ \\
\hline Abnormal shape & $\begin{array}{l}\text { - Salpingitis } \\
\text { - External pressure on oviduct compress- } \\
\text { ing the egg as it forms eg, fluid in } \\
\text { abdomen due to yolk-related peritonitis }\end{array}$ \\
\hline
\end{tabular}

Yolk-related peritonitis occurs when yolk is deposited into the abdomen rather than passing down the oviduct. The infundibulum in ostriches is a large, delicate membrane that moves up and surrounds a developing ovum. Any inflammation of the oviduct or infundibulum can result in this process not proceeding normally, and the ovum fails to enter the oviduct. Alternatively, the ovum does enter the oviduct, but retropulsion pushes it cranially and out through the infundibulum. The yolk constituting the ovum provokes a peritoneal inflammatory response, producing large volumes of fluid as a result. Affected hens usually have a history of having been good layers that suddenly stop laying eggs or may produce abnormally shaped eggs. Examination shows distension of the abdomen and a flaccid, enlarged vent. A fluid wave can be balloted on cloacal examination, and ultrasound can detect the fluid accumulation. Hematology reflects a marked leukocytosis. Abdominocentesis, performed with an appropriate catheter (eg, $14 \mathrm{ga}$ in an ostrich) on the flank behind the last rib, reveals a yellowish pink fluid. Early or mild cases may respond to antibiotic and anti-inflammatory therapy, but severe cases may require a flank laparotomy to allow drainage of the fluid and lavage to remove yolk material.

Salpingitis can be due to infection (ascending or descending) or bruising following excessive or difficult egg laying. The bird may show any or all of the following signs: cessation of egg production, cloacal discharge, stress lines on eggs (Fig 41.24), increased embryonic mortality, or signs of ill health. Physical examination and laboratory work-up other than a leukocytosis may be unrewarding. Ultrasonography may reveal caseous mate- 


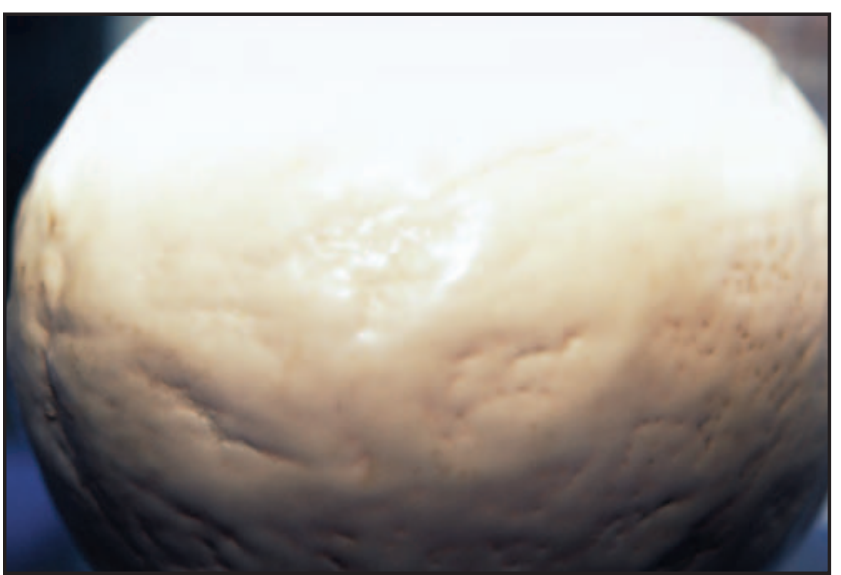

Fig 41.24 | Stress lines in an ostrich egg from a hen with salpingitis.

rial and fluid within the oviduct, and an oviduct swab may detect the presence of infection and/or inflammation. Antibiotic therapy should be based on culture and sensitivity, and cases with caseous exudate in the oviduct may require the surgical insertion of a Foley catheter into the magnum or isthmus, followed by normograde flushing to expel debris from the oviduct. ${ }^{33}$

Two ostriches with caseous salpingitis were treated with dinoprost tromethamine, a prostaglandin $\mathrm{PGF}_{2 \alpha}$ salt. $^{\mathrm{d}}$ In both cases, the hens were treated with one 5-mg injection. This was followed within 15 minutes by signs of abdominal discomfort (grunting, straining to urinate and defecate, and restlessness), which lasted for approximately 1 hour. Systemic antibiotic therapy was started and given for 1 week after the prostaglandin. Follow-up ultrasonography 48 hours after the prostaglandin showed that the caseous material within the oviduct had gone, and both hens went on to breed successfully within 6 months (Doneley, personal observation). Although this treatment caused considerable discomfort to the patients, with further investigation it may prove to be a viable alternative to surgery. Ed Note: Try PGE ${ }_{10 r 2}$ either applied to cervix or injected into the oviduct to reduce pain and dilate the cervix. This probably is a more expensive therapy then owners would prefer, but it is a more bumane therapy.

Egg binding is uncommon in ratites, or at least uncommonly diagnosed. With the exception of the kiwi, the size of ratite eggs relative to body size is quite small, so the clinical signs associated with egg binding in other species are not seen in ratites. The only clinical sign may be cessation of egg laying. In smaller ratites, the egg may be palpable in the abdomen, but in larger ratites (or cases where the diagnosis is uncertain), ultrasound is usually diagnostic. Conservative treatment with oxytocin and calcium may resolve some cases, but surgery may be required.

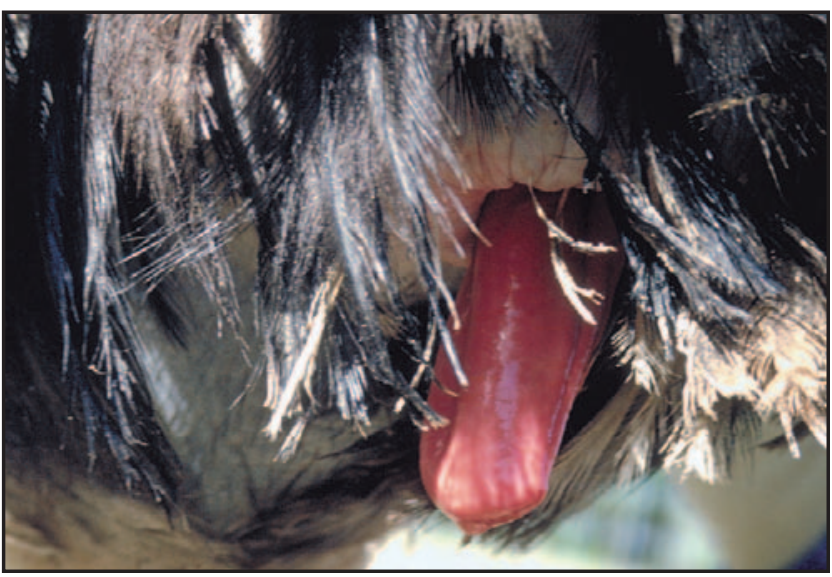

Fig 41.25 | Prolapsed phallus, mature ostrich cock.

Although uncommon, prolapse of a large portion of the oviduct of mature egg-producing hens can occur. The prognosis for these hens is generally very poor. Treatment must be instigated as soon as possible after the prolapse and requires general anesthesia and careful replacement of the oviduct, followed by a purse-string suture. If the oviduct has been prolapsed for a significant time, tissue viability often is compromised. Antibiotics and anti-inflammatory drugs are given postoperatively. An ultrasound examination is strongly recommended to detect the possibility of the presence of egg retention or other oviduct pathology (Black, personal communication).

In contrast to oviductal prolapses, an apparent cloacal prolapse is seen in immature ostrich hens aged 12 to 20 months before egg production begins. The immature oviduct secretes an albumen-like fluid as it matures. This normally drains freely into the cloaca and is occasionally noticed by observant farmers. In some birds, a persistent membrane covers the opening of the oviduct into the urodeum. This prevents the fluid from draining. As pressure builds up behind this membrane, a balloon-like structure arising from the urodeum forms and eventually bulges out through the vent, appearing as a prolapse 5 to $10 \mathrm{~cm}$ in diameter. Once the diagnosis is confirmed by cloacal examination, the membrane can be incised with a scalpel and the newly created opening widened manually. This allows the fluid in the oviduct to drain and the problem resolves. Prophylactic antibiotic coverage is at the clinician's discretion.

\section{Disorders of the Male Reproductive Tract}

Phallic prolapse can occur in both immature and mature birds (Fig 41.25). Immature birds attempting to mate inappropriately can damage the phallus, leading to a prolapse. As well as the treatment described below, these birds need to be isolated from other birds to allow them time to sexually mature. Mature birds can develop 
a phallic prolapse following trauma or infection of the phallus and its surrounding structures or because of excessive sexual activity. Occasionally, an unhealthy bird will develop a prolapse due to generalized weakness.

Purse-string suture techniques should be a treatment of last resort in these birds. If the phallus is not traumatized or becoming desiccated, conservative treatment is usually sufficient. Isolation and sexual rest, combined with lubrication and frequent replacement of the phallus into the cloaca, may be sufficient. Anti-inflammatory and antibiotic therapy may be useful adjuncts. If the phallus is suffering significant trauma or drying out, a temporary purse-string suture combined with anti-inflammatory and antibiotic therapy may be required.

\section{Infertility}

The consistent production of infertile ("clear") eggs requires a thorough veterinary investigation. Before beginning such an investigation, it is vital to review records and procedures. All eggs that fail to develop or hatch should be necropsied as described earlier. Numerous cases of assumed infertility have subsequently been shown to be early, or even mid-term, embryonic deaths.

Possible causes for true infertility include the following:

- Incompatibility: Some birds simply are not compatible and will not mate. Occasionally, if birds are run as trios or in colonies, a dominant hen may prevent mating with other hens by driving them away from the cock.

- Immaturity: Many farmers have unrealistic expectations of when they can expect their birds to perform. As hens generally mature earlier than cocks, egg production may occur before the cocks are physically capable of mating.

- Environmental disturbance: If birds are feeling threatened by disturbances in their immediate environment (such as construction work, traffic, dogs), they may show little interest in mating. The presence of another cock bird in the adjoining enclosure may trigger territorial behavior and inhibit mating.

- Nutrition: Excessively fat or excessively thin birds often show little interest in mating. Imbalances in vitamin and mineral content of the diet may cause decreased spermatogenesis.

- Poor health: Sick or injured birds may not mate.

- Time of season: As most ratites are seasonal breeders and the hens come into breeding condition before the cocks, the first few eggs of a breeding season may be infertile.

- True male infertility: This is uncommon and can be assessed by semen collection and evaluation.

\section{MISCELLANEOUS DISEASES}

Erysipelas is occasionally diagnosed in emus in Australia and the United States. E. rbusiopathiae is transmitted by ingestion of contaminated soil and through skin lacerations. Typically, affected birds die with few clinical signs other than a short period of depression. Necropsy shows hepatomegaly, splenomegaly and petechial hemorrhage on serosal surfaces of the viscera. Culture of $E$. $r h u$ siopathiae from the liver, spleen or heart blood is diagnostic. Affected birds can be treated with penicillin or quinolone antibiotics. The turkey vaccine can be used in susceptible birds at 6 weeks, 20 weeks and 40 weeks..$^{58}$

Salmonellosis has been reported in ostriches, emus, kiwis and rheas. Salmonella pullorum, S. typhimurium and $S$. arizonae have been isolated, with both vertical and horizontal transmission possible. Carrier birds, rodents, freeliving birds and mammals can act as reservoirs for the infection. Clinical signs can include embryonic and neonatal mortality, and depression, diarrhea and sudden death in juvenile and adult birds. Antibiotic therapy (eg, quinolones) can suppress clinical signs but may create chronic carriers. Good biosecurity measures are necessary to both prevent infection and to limit its spread..$^{58}$

Anthrax, caused by Bacillus anthracis, has been diagnosed in ostriches in South Africa. Spores lying dormant in the soil for many years act as the reservoir for infection. Death occurs acutely with few clinical signs.

Demonstration of the characteristic organism in blood smears is diagnostic. There is no recommended treatment. Carcasses should be disposed of by incineration (Editors Note: Burned Spores are liberated and this may not be an ideal practice) or deep burial. An inactivated vaccine is available. ${ }^{58}$

A necrotizing typhlitis, associated with a mixed spirochete and trichomonad-like protozoan, has been reported in rheas. Juveniles older than 1 month are susceptible, with affected birds showing depression and anorexia. The mortality rate may exceed $50 \%$. Necropsy shows distension and hyperemia of the ceca and colon, with fibronecrotic and pseudomembranous changes. Oral metronidazole and parenteral lincomycin reduce mortality in affected flocks.

Plasmodium spp. have been reported in ostriches in Africa, in rheas in Brazil and in emus in North America. Leukocytozoon spp. are considered common in juvenile ostriches in South Africa. ${ }^{34}$ An as-yet-unidentified Babesia sp. has been isolated from North Island brown kiwis in New Zealand and is considered common in wild populations. A Hepatozoon sp. also has been recovered from the same birds. ${ }^{38,50}$ Affected birds of all species show weakness and weight loss, with a regenerative anemia. Treatment of 
avian malaria is discussed in other textbooks.

Aegyptianella pullorum, a rickettsia, is transmitted by argasid ticks in Africa and causes anemia, pyrexia and death in ostrich chicks. Diagnosis is by detection of the organism in erythrocytes. Treatment with tetracyclines is usually curative..$^{34}$

\section{Anesthesia and Surgery of Ratites}

The size and potential danger of many ratites present unique challenges to the avian anesthetist. An anesthetic regime must offer a quick, safe and minimally stressful induction, consistent maintenance and a rapid recovery, while at the same time minimizing the exposure of the operator to risk of injury from the patient. To these ends, a number of anesthetic regimes have been devised.

Masking with isoflurane at $5 \%$ at oxygen flow rates of 2 to 4 liters per minute can usually safely and easily induce ratites weighing less than 15 to $20 \mathrm{~kg}$. Once induced the bird can be intubated and maintained on 2 to $3 \%$ isoflurane at 1 to 2 liters per minute. Assisted ventilation may be advantageous in longer procedures. Recovery is usually rapid, and the bird should be manually restrained or wrapped in a towel until fully conscious.

Larger ratites will require injectable induction, followed by intubation and gaseous maintenance. The following are some suggested protocols:

- Sedation with intramuscular azaperone (0.5-2.0 $\mathrm{mg} / \mathrm{kg}$ ), followed 10 to 15 minutes later with an intravenous combination of ketamine $(8-10 \mathrm{mg} / \mathrm{kg})$ and diazepam (0.2-0.4 $\mathrm{mg} / \mathrm{kg})$, mixed in the same syringe and given as a bolus

- Tiletamine-zolazepam $(2-8 \mathrm{mg} / \mathrm{kg})$ intravenously ${ }^{51}$

- Alphaxalone/alphadolone (2.2-4.8 mg/kg) intravenously ${ }^{51}$

- Ketamine ( $5 \mathrm{mg} / \mathrm{kg}$ IM or $2.2 \mathrm{mg} / \mathrm{kg} \mathrm{IV}$ ) plus xylazine $(1 \mathrm{mg} / \mathrm{kg} \text { IM or } 0.25 \mathrm{mg} / \mathrm{kg} \mathrm{IV})^{20}$

- Medetomidine (0.26-0.54 mg/kg IM) has been used in cassowaries for sedation and restraint, ${ }^{70}$ and at 0.1 $\mathrm{mg} / \mathrm{kg}$ for sedation of ostriches ${ }^{20}$

It is important when anesthetizing large ratites to ensure that adequate padding is placed under the pelvis and femur to avoid peroneal paralysis. If possible, the bird should be positioned in sternal recumbency to assist ventilation. If lateral or dorsal recumbency is necessary, assisted ventilation will be required at a rate of 6 to 12 breaths per minute. The expandable pouch in the trachea of emus must be bandaged during this procedure.
Analgesia and intravenous fluid administration will contribute toward a smooth course of anesthesia. Opioids such as butorphanol or NSAIDs, such as flunixin, carprofen and meloxicam, have been used in ratites. Care should be taken to avoid both hypothermia and hyperthermia, and regular assessment of cloacal temperature is useful in long procedures.

For anesthetic recovery, the bird should be placed into sternal recumbency. This is a potentially dangerous time for the patient. Birds startled by loud noises or other stimuli may rear up and fall over backward, causing themselves injury and even killing themselves. For this reason they should be placed in a quiet, dark area and movement kept to a minimum. Placing them in a crate or horse trailer or surrounding them with hay bales can prevent them from rolling into lateral recumbency. Ostriches should be hooded, and remain hooded, until they can hold their heads upright unaided.

\section{ABDOMINAL SURGERIES ${ }^{5}$}

\section{Chicks}

Most laparotomies in small ostrich chicks are performed with the bird in dorsal recumbency.

The approach for yolk sac surgery or exploration of the abdominal cavity is via a ventral midline incision. In yolk sac excisional surgery, an elliptical incision incorporating the umbilicus is utilized. Immediately below the skin is an extremely thin abdominal lining. This lining consists of a connective tissue layer with a black-pigmented peritoneum immediately below. Care should be taken in incising these layers, as the dilated yolk sac can be very close or even adhered to the peritoneum. Once the abdomen is exposed, the incision may have to be lengthened to enable the yolk sac to be exteriorized. Again, care must be taken as the yolk sac may rupture if excessive pressure is applied to it. The vitelline duct and blood vessels are ligated between the yolk sac and duodenum, and the yolk sac is removed. The abdomen can be irrigated using a warmed saline solution, and a small amount of aqueous antibiotic can be infused (eg, amoxicillin or ampicillin). This is particularly important if the yolk sac has been ruptured either before or during the surgery. Occasionally the yolk sac may be very inflamed and adhered to the abdominal wall or intestine. Careful dissection is necessary to remove all yolk sac tissue without significant damage to the intestinal serosa. The abdominal incision is closed in two layers: muscle and skin.

A proventriculotomy is performed via an incision over the proventriculus on the left ventral abdomen. This incision normally is sited within the left abdominal pterylae. If the incision is properly sited, the surgical 
area is isolated from the rest of the abdomen by fascia and air sac lining, and suturing of the proventriculus to the abdominal wall is unnecessary. If the incision is positioned too far back toward the posterior abdomen, the intestines can be directly visualized and accessed. This area should then be closed by suturing before the proventriculus is entered to avoid contamination of the abdomen by spillage of proventricular contents. The relatively thin proventricular wall is located just below the thin ventral abdominal wall. The proventriculus and the ventriculus can both be accessed via this approach.

The ventriculus should be approached via a proventriculotomy rather than directly incising the ventriculus. Any free fluid in the proventriculus can be aspirated and then the impacting material or foreign bodies can be removed. The closure is in three layers: proventriculus, abdominal wall and skin. The proventricular closure is achieved using a continuous Lembert-type suture pattern using absorbable synthetic suture material. The abdominal wall is closed with similar suture material. The skin closure can be done with absorbable or nonabsorbable material.

\section{Older Chicks and Adults}

Abdominal surgery on older ostrich chicks and adults is usually performed with the birds in lateral recumbency, lying on their right side.

Entry into the abdominal cavity is via an incision through the left abdominal wall in the flank apterylae. The position and orientation of this incision will be determined by the nature of the condition leading to the laparotomy. For example, most egg yolk peritonitis surgeries are performed via a vertical incision about halfway between the back of the left thigh and the posterior margin of the abdomen. In some instances, a horizontal incision may be used (eg, to gain greater oviduct exposure), or even a T-type incision combining both can be used. The horizontal incision usually results in greater hemorrhage and is subjected to much greater tension on closure. The skin is incised to reveal two muscle/fascial layers directly below (there is very little subcutaneous tissue). Between the second muscle/fascial layer and the peritoneum is a variable and sometimes excessive fat layer. The peritoneum is opened by puncture using blunt-pointed scissors. The peritoneum is pigmented (gray color of varying darkness) and very tough.

With yolk-related peritonitis, significant yolk-stained fluid can be present in the abdomen. This fluid will immediately begin draining through the surgical opening, often under pressure because of the bird's positioning. This fluid can be aspirated or allowed to passively drain. Tilting the bird's abdomen downward facilitates drainage. The abdomen can be manually explored to detect the presence of inspissated yolks, retained eggs or neoplasia. The aim of the surgery is to extensively flush the abdomen until all yolk debris has been removed. This can be a very difficult exercise in view of the size of the abdomen, the length of intestine present and the relatively difficult access. The abdomen is flushed with $0.25 \%$ Betadine in saline until no further debris is found and the fluid escaping the abdomen becomes clear. This can take up to 12 to 14 liters of fluid. A final liter of saline and antibiotic is instilled into the abdomen, and $250 \mathrm{ml}$ of this solution is usually infused into the oviduct lumen via a needle through the anterior oviduct.

The oviduct is visualized to detect any pathology such as edema, inflammation or neoplasia. The entire length is palpated to detect any blockages or retained eggs. The infundibulum also is visually inspected. It is imperative to avoid much handling of the infundibulum. This is a highly motile, thin and delicate structure, which is easily torn, bruised or inflamed. It is also important to ensure that the infundibulum is kept well away from the incision site during suturing of the peritoneum.

The closure of the abdomen is normally done in four or five layers. The peritoneum is closed with synthetic absorbable suture in a simple continuous pattern. The two muscle/fascial layers are then closed either separately or as one layer using similar material. A subcutaneous suture may then be done using a lighter absorbable suture. The skin is usually closed with non-absorbable suture. This skin closure is an interrupted pattern of simple or mattress sutures.

The prognosis associated with this procedure is influenced by the length of time the condition has been present and the degree of damage found in the abdomen. Prognosis for survival is excellent, but prognosis for future productivity is guarded, with expected success rates, with experience, of about $66 \%$.

Severe salpingitis cases may best be treated surgically. An intrauterine infusion of an appropriate antibiotic saline solution is employed via a needle or Foley catheter introduced into the proximal oviduct (magnum). A volume of 250 to $1000 \mathrm{ml}$ is used, depending on the extent and nature of the infection. Care must be taken to avoid retrograde passage of fluid back into the abdomen via the infundibulum.

Some retained eggs may be accessed via the cloaca and removed manually while the bird is anesthetized. Eggs higher in the oviduct can be removed only surgically. The site of the retention can be previously ascertained by an ultrasound examination. Access to the lower oviduct is difficult. The oviduct is very friable and has 
poor suture-holding properties. After egg removal from the incised oviduct, the wall is sutured carefully using synthetic absorbable suture material in a continuous Lembert-type pattern.

Proventriculotomy surgery in older birds is done in a fashion similar to that of chicks with the amount of abdominal fat being the major difference. It is not uncommon to encounter up to $15 \mathrm{~cm}$ of abdominal fat during the surgical approach. This makes access limited in some cases and creates some difficulty for easy suturing.

Occasionally, a decision may be made to perform the laparotomy with the bird in dorsal recumbency and the incision made on the ventral abdomen. This is rarely done, but may be required to access some intestinal problems (especially those involving the small intestine). In these cases, it is necessary to slightly extend the legs to avoid potential nerve and muscle injury at the level of the upper thigh during the procedure.

Intestinal volvulus is not uncommon in ostriches and is especially associated with diet changes. Some cases will occur without any obvious predisposing trigger. Gutpenetrating injuries also can occur after ingestion of sharp objects such as sticks, nails and wire. Diagnosis is based on clinical signs (anorexia, depression, abdominal pain, lack of fecal passage), abdominal paracentesis, hematology and possible radiology. Ostriches appear to have very little tolerance to musculoskeletal pain, but show remarkable tolerance to visceral and abdominal pain. It is possible that birds with significant peritonitis and avascular necrosis of a section of gut may show only mild symptoms of abdominal pain.

The laparotomy site is usually the left lateral abdomen, as described previously. A decision to access the abdomen via a midline approach may be made if the problem is suspected in the upper small intestine or if there is insufficient access via the lateral route. If nonviable intestine is found requiring resection and anastomosis, a poor prognosis should be given. The large intestinal wall is extremely thin and very difficult to suture successfully. Peritoneal lavage using saline and a saline/antibiotic solution is necessary in all cases, as invariably a septic peritonitis is present. If surgical intervention is early enough, it is possible to correct a volvulus or intussusception without further complications, provided gut viability is present.

\section{Postoperative Care}

Apart from cases of yolk sac excision or egg yolk peritonitis, most laparotomy cases in ostriches do not require intensive postoperative care.

Chicks recovering from yolk sac excisional surgery require close monitoring, heat support and frequent tube feeding. The tube feeding may be necessary for only 24 hours or may be required for several days until the chick becomes self-sufficient and is regularly gaining weight.

Most laparotomy cases require immediate postoperative antibiotics. The final choice of antibiotics will be determined by any culture/sensitivity testing previously carried out. Tube feeding in some chicks also may be required during the postoperative period. Suture removal, if necessary, is done at a minimum of 2 weeks after surgery.

\section{SKIN LACERATIONS}

Generally, lacerations of the skin of the neck, body and upper thighs heal very well once sutured. Some neck lacerations may heal well with minimal intervention. Lower limb wounds are less rewarding due to tension and secondary infection. If possible, administration of general anesthesia to recently traumatized birds is avoided. Local anesthesia, if possible, is safer. If the trauma is too severe for the use of local anesthesia, it is preferable to determine the status of the bird by blood sampling and evaluating the full blood examination and biochemistry profile. This should be combined with immediate stabilization of the patient using intravenous fluids, analgesics, and antibiotics.

Traumatic penetrating wounds to joints and tendon sheaths should be treated vigorously using local and systemic antibiotics. If infection is suspected, culture and sensitivity testing is essential. Joint lavage will be necessary if fistulae and significant joint infection are already present.

\section{ORTHOPEDICS}

The likelihood of the veterinary surgeon being requested to perform orthopedic procedures on ostriches is now quite low. Severe leg fractures or luxations usually result in a decision to euthanize the bird. Most cases of beak trauma can be handled medically. It is quite surprising to see ostriches suffer very little setback, even with major beak trauma resulting in significant defects. Wing fractures will commonly repair after simple taping of the wing back into correct position. This is best achieved by taping wing feathers to body feathers at several sites. This avoids the stress of complete body wrap taping of wings. Severe wing fractures can be handled by amputation if necessary.

\section{EYES}

Surgical repair of eyelid lacerations is common and usually rewarding.

Eyelid closure to treat corneal ulcers and keratitis can be 
performed as described in many textbooks, and may even be done using local anesthesia. The strength of the eyelid muscles is surprising and requires the placement of quite heavy-gauge suture material.

Enucleation can be performed in chicks. In adults this is a difficult procedure with significant risk of hemorrhage. Adult hens have been reported to not breed following unilateral enucleation. This is not always the case, as some birds, apparently in severe pain prior to enucleation, have bred successfully within weeks of the surgery.

Cataract removal using phacoemulsification techniques has been carried out in Australia and the United States (Fig 41.26). This is becoming less common now that bird values have reduced.

Periorbital abscessation resulting from bacterial sinusitis is relatively common in ostrich chicks. These tend to result in discrete swellings on the skull in a dorsomedial position relative to the eye. After local anesthesia, an incision can be made into this swelling to enable debridement and excision of firm gelatinous-like pus. Local irrigation with appropriate antibiotics combined with systemic antibiotic therapy after culture and sensitivity testing are needed to effect a normally uneventful recovery.

\section{Acknowledgements}

I thank the following people for their invaluable contributions to this work. Doug Black (Ostrich Health Consultant, Moama, New South Wales) gave me valuable

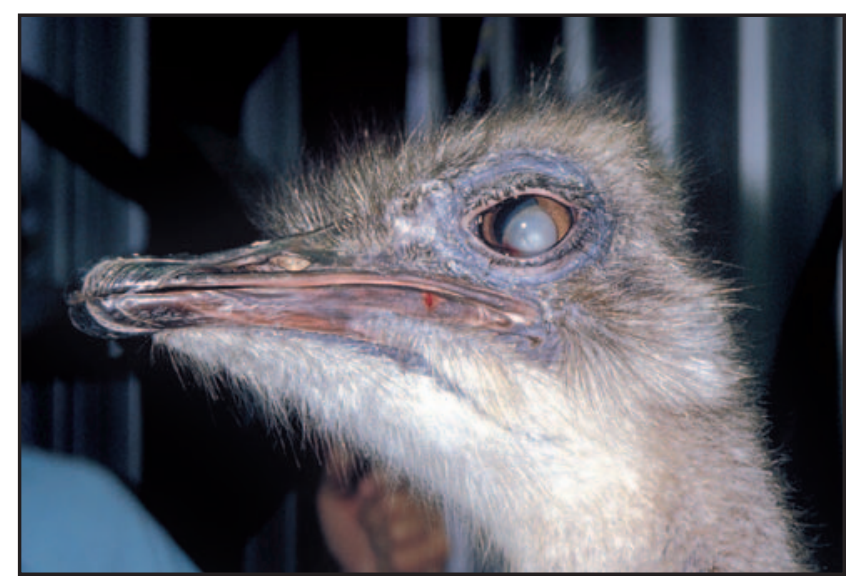

Fig 41.26 | Luxated cataract lens in the ostrich.

advice about writing this chapter, adding to the advice on ostriches he has given me over the last 10 years. Richard Jakob-Hoff (Auckland Zoo, New Zealand) and Hugh Robertson (Science and Research Unit, Department of Conservation, New Zealand) provided much of the information on kiwis, making unique information widely available for the first time. Dale Smith (Ontario Veterinary College, University of Guelph, Canada) discussed with me many aspects of this chapter.

\section{Products Mentioned in the Text}

a. Enilconazole wash, Imaverol ${ }^{\circledast}$, Coopers Australia, North Ryde, NSW www.coopersanimalhealth.com.au

b. Toltrazuril, Baycox ${ }^{\circledast}$, Bayer Australia, Pymble, NSW, www.baycox.com

c. Enilconazole smoke bombs, Clinafarm, Janssen, Sterwin, Belgium

d. Dinoprost tromathamine (prostaglandin PGF2a) Lutalyse, Pharmacia

Upjohn, Rydalmere, NSW, www.lutalyse.com

e. Mazuri, www.mazuri.com

\section{References and Suggested Reading}

1. Angel CR, Scheider SE, Sell JL: Ratite nutrition. In Tully TN Shane SM: Ratite Management, Medicine and Surgery. Malabar, FL, Krieger Publishing, 1996, pp 11-30.

2. Badley AR: Improved assessment of the fertility and development of ostrich eggs by more efficient candling. In Deeming, DC (ed) Improving Our Understanding of Ratites in a Farming Environment Manchester, UK, 1996, pp 146148

3. Bezuidenhout AJ: Anatomy. In Deeming DC (ed): The Ostrich Biology, Production and Health. Oxon, UK, CABI Publishing, 1999, pp 13-49.

4. Black DG: Ostrich examination Post-Grad Comm Vet Sci, University of Sydney. Sydney, Australia, 1995.

5. Black DG: Common medical and surgical conditions of ostriches. In The Basics of Avian Medicine. Proc 2789, Post Grad Foundation Vet Sci, University of Sydney, 1996, pp 243-258.
6. Black DG: Genetic, nutritional and management effects on ostrich hide quality. Proc Assoc Avian Vet Aust Comm, 1999, pp 43-51.

7. Black DG: Ostrich flock health Sem Avian Exotic Pet Med 10(3) 117-130, 2001

8. Blake JP: Nutritional recommendations for emus. In Deeming, DC (ed): Improving Our Understanding of Ratites in a Farming Environment. Manchester, UK, 1996, pp 109-110.

9. Blignaut A, et al: Antibody production in ostriches in response to vaccination with La Sota Newcastle disease virus vaccines. Proc $2^{\text {nd }}$ Intl Ratite Cong, South Africa, 1998, pp 199-200.

10. Boardman WSJ: Suspected biotin deficiency in North Island brown kiwi. Kokako Bull Wildlife Soc New Zeal Vet Assoc 1(1):5, 1994

11. Brand $Z$, et al: The effect of preheating of ostrich eggs prior to storage and setting in commercial hatcheries. Proc $2^{\text {nd }}$ Intl Ratite Cong, South Africa, 1998, pp 152-154.

12. Button K: Ostrich incubation practices and necropsy technique. Proc Aust Ostrich Assoc, Post Grad Comm Vet Sci, University of Sydney, 1993 pp 69-72

13. Button C, Kabay M, Rawlin G: Ostrich fading syndrome in Australia. In Deeming, DC (ed): Improving Our Understanding of Ratites in a Farming Environment. Manchester, UK, 1996, pp 35-38.

14. Calder WA: The kiwi and its egg. In Fuller E (ed): Kiwis: A Monograph of the Family Apterygidae. Auckland, NZ, SeTo Publishing, 1990, pp 155-171.

15. Cilliers SC: Feedstuff evaluation, metabolisable energy and amino acid requirements for maintenance and growth in ostriches. Proc $2^{\text {nd }}$ Intl Ratite Cong, South Africa, 1998, pp 12-23.

16. Cilliers SC, Angel CR: Basic concepts and recent advances in digestion and nutrition. In Deeming DC (ed): The Ostrich: Biology, Production and Health. Oxon, UK, CABI Publishing, 1999, pp 105-128.

17. Cloete SWP, van Schalkwyk SJ, Brand Z: Ostrich breeding: Progress towards a scientifically based strategy. Proc $2^{\text {nd }}$ Intl Ratite Cong, South Africa, 1998, pp 55-62.

18. Cockrem J: Diet and nutrition of captive kiwi. In Billing $\mathrm{T}$ (ed): Proc New Zealand Conservation
Management Group Kiwi Workshop, Auckland Zoo, 1998, pp 69-73.

19. Colbourne R: Operation nest egg. In Billing $\mathrm{T}$ (ed): Proc New Zealand Conservation Management Group Kiwi Workshop, Auckland Zoo, 1998, pp 41-46.

20. Cornick-Seahorn JL: Anesthesiology of ratites. In Tully TN, Shane SM: Ratite Management, Medicine and Surgery. Malabar, FL, Krieger Publishing, 1996, pp 7994

21. Craig TM, Diamond PL: Parasites of ratites. In Tully TN, Shane SM: Ratite Management, Medicine and Surgery. Malabar, FL, Krieger Publishing, 1996, pp 115-126.

22. Deeming DC, Ar A: Factors affecting the success of commercial incubation. In Deeming DC (ed): The Ostrich: Biology, Production and Health. Oxon, UK, CABI Publishing, 1999, pp 159-190.

23. Deeming DC, Bubier NE: Behavior in natural and captive environments. In Deeming DC (ed): The Ostrich: Biology, Production and Health. Oxon, UK, CABI Publishing, 1999, pp 83-104

24. Deeming DC, et al: A review of recent work on the Behavior of 
young ostrich chicks with respect to feeding. In Deeming, DC (ed): Improving our Understanding of Ratites in a Farming Environment.

Manchester, UK, 1996, pp 20-21.

25. Doneley RJT: Principles of incubation. Proc Assoc Avian Vet Aust Comm, 1996, pp 155-167.

26. Doneley RJT: Semen collection in the ostrich. Proc Aust Ostrich Assoc, 1996.

27. Doneley RJT: Flock health schemes and preventative medicine: The veterinarian's view. Proc Aust Ostrich Assoc, 1996.

28. Doneley RJT: Ostrich paediatrics Proc Assoc Avian Vet Aust Comm, 1997 pp 1-20.

29. Doneley RJT, et al: Mycobacterial infection in an ostrich. Aust Vet $\mathrm{J}$ 77(6):368-370, 1999.

30. Elston JJ, Beck MM, Scheideler SE: Behavioral analysis of emu chicks and breeding adults. Proc $2^{\text {nd }}$ Intl Ratite Cong, South Africa, 1998, pp 177-180.

31. Fowler ME: Anatomy. In Tully TN, Shane SM: Ratite Management, Medicine and Surgery. Malabar, FL, Krieger Publishing, 1996, pp 1-10.

32. Fudge A: Clinical hematology and chemistry of ratites. In Tully TN, Shane SM: Ratite Management, Medicine and Surgery. Malabar, FL, Krieger Publishing, 1996, pp 105-114.

33. Hicks-Alldredge KD: Reproduction. In Tully TN, Shane SM: Ratite Management, Medicine and Surgery. Malabar, FL, Krieger Publishing, 1996, pp 47-57.

34. Huchzermeyer FW: Diseases of ostriches and other ratites. Agriculture Research Council, Onderstepoort, Republic of South Africa, 1998.

35. Huchzermeyer FW: Veterinary problems. In Deeming DC (ed): The Ostrich: Biology, Production and Health. Oxon, UK, CABI Publishing, 1999, pp 293-320.

36. Irons PC, et al: Semen collection and evaluation in the ostrich. In Deeming DC (ed): Improving Our Understanding of Ratites in a Farming Environment. Manchester, UK, 1996, pp 157. 159.

37. Jakob-Hoff R, Buchan B, Boyland M: Kiwi coccidia: North Island survey results. Kokako Bull Wildlife Soc New Zealand Vet Assoc 6(1):3-5, 1999.

38. Jakob-Hoff R, Twentyman C, Buchan B: Clinical features asso- ciated with a haemoparasite of the North Island Kiwi. Proc Joint Conf Wildlife Disease Assoc and Wildlife Soc, NZ Vet Assoc, 2000, pp 23-24.

39. Jakob-Hoff R: Establishing a health profile for the North Island brown kiwi (Apteryx australis mantelli). Proc Intl Joint Conf World Assoc Wildlife Vet, Wildlife Dis Assoc, Aust Assoc Vet Conservation Biologists and Wildlife Soc, NZ Vet Assoc, 2001 pp 135-140.

40. Jensen J, Johnson H: Husbandry and medical management of ostriches, emus and rheas. Wildlife and Exotic Animal Teleconsultants, Texas, USA, 1992.

41. Lábaque C, Navarro JL, Martella MB: Chick adoption and subsequent survival in the greater rhea. Proc $2^{\text {nd }}$ Intl Ratite Cong, South Africa, 1998, pp 175-176.

42. Lambrechts H, Huchzermeyer FW, Swart, D: Integrating ostrich Behavior in commercial farming systems. Proc $2^{\text {nd }}$ Intl Ratite Cong, South Africa, 1998, pp 167-169.

43. Love SCJ, Gill HS: Aspergillosis in ostriches: Epidemiology, treatment and diagnosis. Proc $5^{\text {th }}$ Aus Ostrich Assoc Conf, 1995, pp 5359.

44. Malecki IA, Martin GB, Lindsay DR: Semen collection and production of spermatozoa in the male emu. In Deeming, DC (ed): Improving Our Understanding of Ratites in a Farming Environment. Manchester, UK, 1996, pp 159-160.

45. Mouser D: Restraint and handling of the emu. In Deeming, DC (ed): Improving Our Understanding of Ratites in a Farming Environment. Manchester, UK, 1996, pp 41-45.

46. Navarro JL, et al: Diet of wild greater rheas in an agroecosystem of southern Córdoba (Argentina). In Deeming, DC (ed): Improving Our Understanding of Ratites in a Farming Environment. Manchester, UK, 1996, pp 110-111.

47. O'Malley PJ: An estimate of the nutritional requirements of emus. In Deeming, DC (ed): Improving Our Understanding of Ratites in a Farming Environment. Manchester, UK, 1996, pp 92-108.

48. Peach P: Detection of retroviral infections and their application to the detection of a novel retrovirus isolated in ostriches diagnosed with ostrich fading syndrome. Proc Assoc Avian Vet Aust Comm, 1997, pp 143-149.

49. Peat $\mathrm{N}$ : The incredible kiwi. Random Century in association with Television New Zealand, 1990.

50. Peirce MA, Jakob-Hoff RM, Twentyman C: New species of Apterygidae in New Zealand. J Nat Hist, in press.

51. Perelman B: Health management and veterinary procedures. In Deeming DC (ed): The Ostrich: Biology, Production and Health. Oxon, UK, CABI Publishing, 1999, pp 321-346.

52. Potter MA, Fordham RA, McLennan JA: Reproductive biology of kiwi. In Deeming, DC (ed): Improving Our Understanding of Ratites in a Farming Environment. Manchester, UK, 1996, pp 161-162.

53. Raidal SR, Gill JH, Cross GM: Pox in ostrich chicks. Aust Vet J (73):32-33, 1996.

54. Raines AM: Adenovirus infection in the ostrich (Strutbio camelus). Proc Assoc Avian Vet, 1993, pp 304-312.

55. Romer L: Cassowary Husbandry Manual. Workshop materials, Currumbin Sanctuary

Conservation Unit, Currumbin, Queensland, 1997.

56. Ross EJ, Deeming DC, Dawkins MS: Feeding and vigilance Behavior of breeding ostriches in a farming environment in Britain. In Deeming, DC (ed): Improving Our Understanding of Ratites in a Farming Environment. Manchester, UK, 1996, pp 24-26.

57. Schaetz L, et al: Investigation into the immune response of ostrich chicks (Strutbio camelus) after vaccination against Newcastle disease. Proc 2nd Intl Ratite Cong, South Africa, 1998, pp 195-198.

58. Shane SM, Tully TN: Infectious diseases. In Tully TN, Shane SM: Ratite Management, Medicine and Surgery. Malabar, FL, Krieger Publishing, 1996, pp 127-146.

59. Sharp PJ, et al: Neuroendocrine control of incubation Behavior in the emu. In Deeming, DC (ed): Improving Our Understanding of Ratites in a Farming Environment. Manchester, UK, 1996, pp 162-1163.

60. Soley JT, Groenewald HB:
Reproduction. In Deeming DC (ed): The Ostrich: Biology, Production and Health. Oxon, UK, CABI Publishing, 1999, pp 129-158.

61. Speer BL: Developmental problems in young ratites. In Tully TN, Shane SM: Ratite Management, Medicine and Surgery. Malabar, FL, Krieger Publishing, 1996, pp 147-154.

62. Stewart J: Ratite incubation. Proc Assoc Avian Vet, 1992, pp 358359.

63. Stewart J: Ratites. In Ritchie BW, Harrison GJ, Harrison LR: Avian Medicine: Principles and Application. Brentwood, TN HBD Intl Inc, 1999.

64. Stewart J: Hatchery management in ostrich production. In Tully TN, Shane SM: Ratite

Management, Medicine and Surgery. Malabar, FL, Krieger Publishing, 1996, pp 59-67.

65. Van Schalkwyk SJ, et al: The influence of different disinfection protocols on the hatching performance of ostrich eggs. Proc $2^{\text {nd }}$ Intl Ratite Cong, South Africa, 1998, pp 157-159.

66. Vercoe D: Auckland Zoo native fauna conservation centre breeding program: Achievements for the 2001/2002 season. Unpublished internal report, Auckland Zoo, 2002.

67. Verwoerd DJ: Newcastle Disease in ostriches: A review of experimental data. Proc $2^{\text {nd }}$ Intl Ratite Cong, South Africa, 1998, pp 192-194.

68. Verwoerd DJ, et al: Rearing environments around the world. In Deeming DC (ed): The Ostrich: Biology, Production and Health. Oxon, UK, CABI Publishing, 1999, pp 191-216.

69. Vorster JH, Olivier AJ: Diseases affecting the central nervous system of ostriches in southern Africa. Proc $2^{\text {nd }}$ Intl Ratite Cong, South Africa, 1998, pp 201-204.

70. Westcott DA, Reid KE: Use of medetomidine for capture and restraint of cassowaries (Casuarius casuarius). Aust Vet J 80(3): 150-153, 2002.

71. Williams KM, et al: Growth, sexual development and carcass composition in intact and surgically or hormonally gonadectomised male and female emus. Proc $2^{\text {nd }}$ Intl Ratite Cong, South Africa, 1998, pp 75-80. 


\section{Management of Captive}

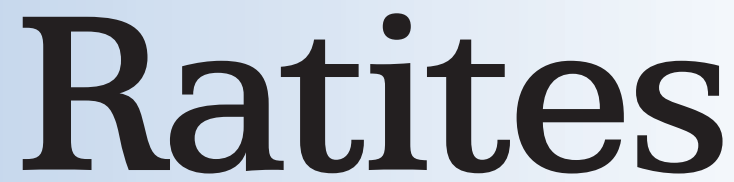

\section{BOB DONELEY, BVSc, FACVSc (Avian Health)}

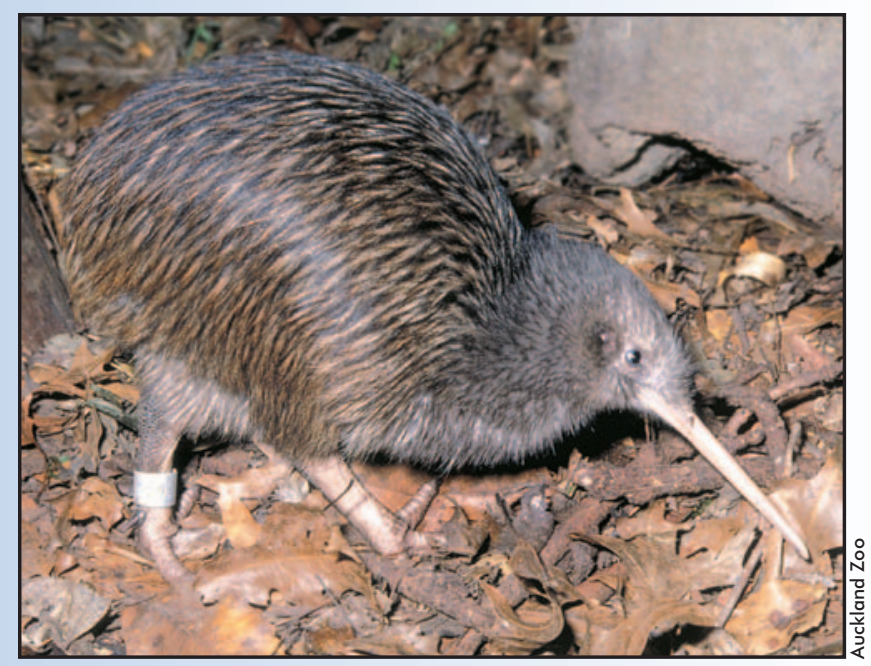

Fig 41.1 | North Island brown kiwi.
Although the demand for veterinary input into the ostrich and emu industries is not as great today as it was in the 1990s, veterinarians are still called on to give advice and to treat ratites of all descriptions. This chapter seeks to present new and updated information on these flightless birds as well as refer the reader to references where more comprehensive information may be found.

\section{Classification}

Ratites are not an order or family of birds. Rather, they are a group of birds sharing similar physical characteristics. They are all flightless birds, with elongated necks and relatively long legs adapted for walking and running (Fig 41.1, Table 41.1). The name ratite comes from the Latin ratis, or raft. This refers to the flattened, raft-like sternum that lacks a keel and ventral musculature an adaptation to their loss of flight. Ratites are naturally found on three continents (Africa, Australia and South America) and two islands (New Guinea and New Zealand), all in the Southern Hemisphere. Nevertheless, commercial development of the ostrich and emu industry is seen on every continent except Antarctica. 
Table 41.1 | Classification of Ratites.

There are 11 species of birds grouped as ratites. They come from four orders and five families.

\begin{tabular}{|l|l|l|l|l|}
\hline Common Name & Order & Samily Subspecies & \multicolumn{2}{|c|}{$\begin{array}{l}\text { S.c. camelus (North African) } \\
\text { S.c. molybdophanes (Somali) } \\
\text { S.c. massaicus (Massai) } \\
\text { S.c. australis (South African) }\end{array}$} \\
\hline Emu & Struthioniformes & Struthionidae & Struthio camelus & - \\
\hline Southern/double-wattled cassowary & Casuariiformes & Casuariidae & Casuarius casuarius & - \\
\hline Bennett's/little cassowary & Casuariiformes & Casuariidae & C. bennetti & - \\
\hline Northern/single-wattled cassowary & Casuariiformes & Casuariidae & C. unappendiculatus & - \\
\hline Greater rhea & Rheiformes & Rheidae & Rhea americana & - \\
\hline Darwin or lesser rhea & Rheiformes & Rheidae & R. pennata (formerly Pterocnemia pennata) & \\
\hline Tokoeka kiwi & Apterygiformes & Apterygidae & Apteryx australis & - \\
\hline Brown kiwi & Apterygiformes & Apterygidae & A. mantelli & - \\
\hline Little spotted kiwi & Apterygiformes & Apterygidae & A. oweni & - \\
\hline Great spotted kiwi & Apterygiformes & Apterygidae & A. haasti & - \\
\hline Rowi kiwi & Apterygiformes & Apterygidae & A. rowi & - \\
\hline
\end{tabular}

Some classifications place the tinamous (Order Tinamiformes; Family Tinamidae), a South American partridge-like bird, in the ratite group. They share some physical characteristics with the ratite groups described above, especially the rhea.

\section{Clinical Anatomy and Physiology}

Ratite anatomy and physiology are similar to other avian species, particularly poultry and psittacines, although there are some clinically significant differences. ${ }^{3,31,34,63}$

\section{INTEGUMENT}

The skin of ratites is thicker than that of many other avian species; consequently, ostrich and emu hides are in demand by the leather industry. Rough handling easily damages the skin, and scarring or bruising detracts from the hide's value. Care must be taken when handling these birds to avoid such damage. Kiwi skin is particularly thick and includes a prominent hypodermis for fat storage (Fig 41.2). Sternal callosities (dermal thickening for weight bearing when in sternal recumbency) often are present over the sternum of ostriches, emus and rheas. A similar condition also frequently is present over the ventral-cranial portion of the pubic bone in the ostrich. The plantar surface of the foot has a dermal pad of packed vertical rods of cornified tissue, with underlying paired, tubular fat bodies for additional padding.

The toenails are blunted in ostriches, but are sharper in other species, especially cassowaries, which can have 12 $\mathrm{cm}$ spikes on the medial toes. Prior to the breeding season, male North Island brown kiwis can store as much as $50 \%$ of their body mass as subcutaneous and intracoelomic fat. The feathers of ratites lack the barbules seen in other species, and so the feather vane does not interlock tightly. This gives ratite feathers a unique hairlike appearance, but also makes them less water-resistant. Contrary to earlier work, the presence of filoplumes has been reported in ostriches. ${ }^{6}$ These slender feathers

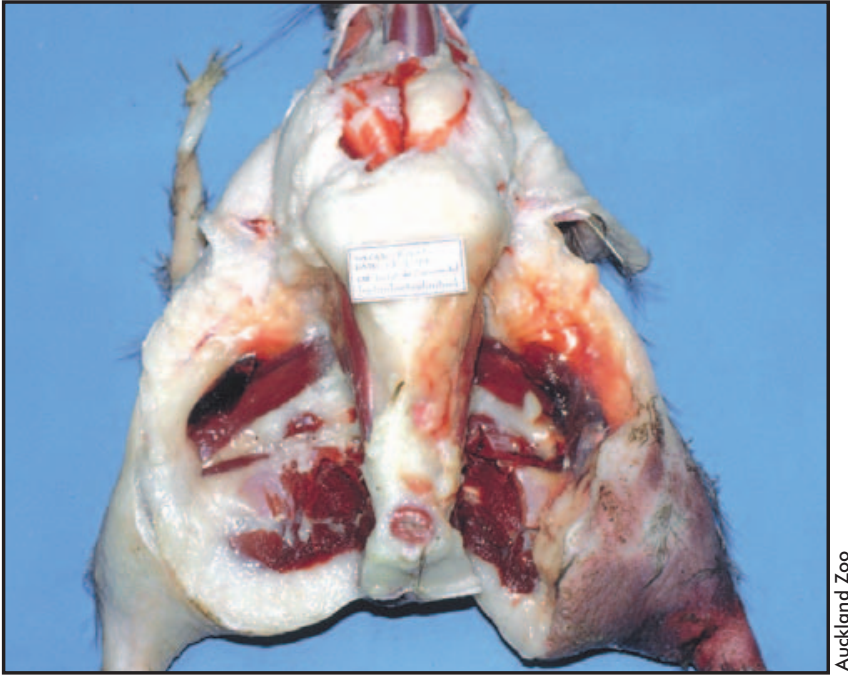

Fig 41.2 | Dissection showing massive deposits of subcutaneous and intracoelomic fat in a male North Island brown kiwi early in the breeding season (September). Ironically, the bird died after being caught in a leg-hold trap set for its mammalian predators.

arise from small follicles alongside the larger follicles of contour feathers. The presence of a large number of filoplumes (and therefore their follicles) can detract from the value of a hide. There may be a genetic factor for these filoplumes, and their presence should be considered a selection criterion for breeding birds. ${ }^{6}$

Thermoregulation in ostriches is achieved by evaporative cooling from the respiratory tract and convection heat loss through the skin. The thighs of the ostrich, normally covered by the wings, are bare of feathers. Contour feathers on the body can be erected or flattened voluntarily. A heat-stressed ostrich will hold its wings away from the body and erect its contour feathers, thus maximizing heat loss from the body (Fig 41.3). Conversely, in 


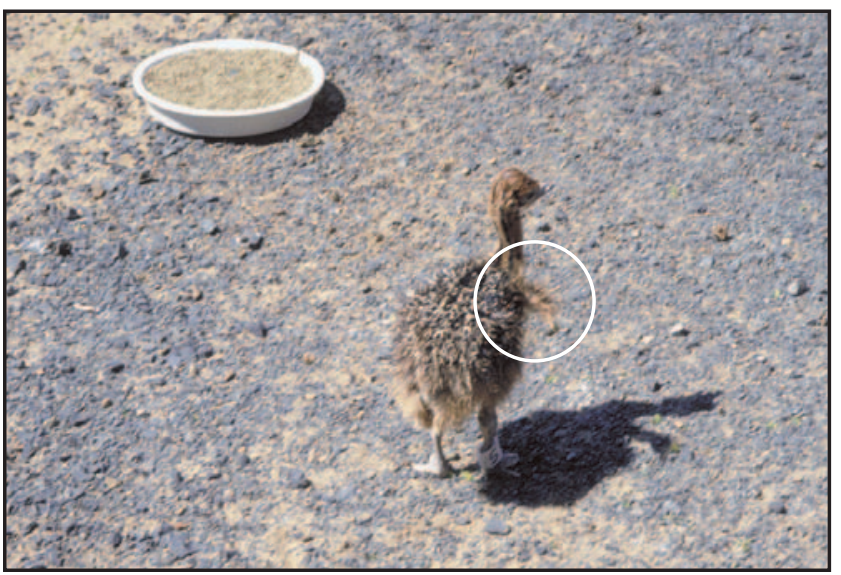

Fig 41.3 | Heat stress in an ostrich chick. Note the raised feathers and the wings held away from the body, thus maximizing heat loss. In these conditions, appetite can be reduced by as much as $40 \%$, with resultant stunting and mortality.

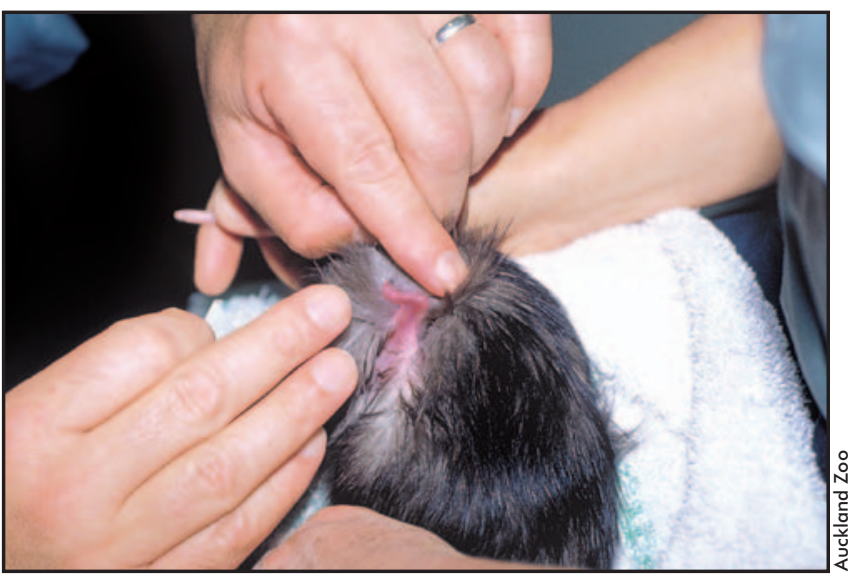

Fig 41.4 | North Island brown kiwi chick showing vestigial wing and axillary apteria (featherless skin), which is the site used to implant subcutaneous identity microchips. cold weather, the ostrich will flatten its feathers and wings against the body, conserving body heat. It may also lie down on its sternum, further reducing the area of skin available for convective heat loss.

\section{MUSCULOSKELETAL SYSTEM}

With the loss of flight, ratites have lost the need for pneumatized bones. Consequently, with the exception of a pneumatized femur in the ostrich and emu, ratite bones are heavier and denser than those of other avian species. In the ostrich, the thoracic girdle (coracoid, clavicle and scapula) has fused, a further adaptation to the loss of flight. Ostriches and rheas have relatively large wings, while the wings of other ratites are comparatively much smaller (Fig 41.4). Ostriches have two toes (digits 3 and 4), the rest have three (digits 2, 3 and 4) all forward, and all toes have four phalanges on each digit.

In the ostrich, emu and rhea, the muscles of economic importance are found on the pelvic limb and along the lumbar vertebrae. This makes these muscles unsuitable for intramuscular injections, as abscesses or scar formation could lead to downgrading or condemnation of the carcass. In all ratites, the reliance on the legs for locomotion means that particular care must be taken that intramuscular injections do not result in pain and lameness. The lack of pectoral muscles on the ventral sternum leaves the muscles alongside the thoracic vertebrae as the area most suitable for injections. Note that care must be taken to minimize hide damage through repeated injections.

\section{DIGESTIVE SYSTEM}

Ratites are primarily herbivorous (although cassowaries are known to eat small mammals), and their digestive tracts are basically similar to those of other herbivorous birds. ${ }^{31}$ The exception is the kiwi, which is primarily insectivorous. ${ }^{18}$ Its anatomic and physiologic differences reflect the evolutionary influences of the forages available in their natural habitat.

The esophagus lies on the right side of the neck. It contains numerous longitudinal rugae that allow expansion when a bolus of food is swallowed. The ostrich cock, when displaying territorial behavior or seeking to attract a mate, inflates the esophagus with air and then expels it with a loud "boom". There is no crop in any of the ratites, and the esophagus opens directly into the proventriculus. There is no esophageal/proventricular sphincter; therefore, regurgitation of proventricular contents can be of concern during anesthesia.

In the ostrich, the large, thin-walled proventriculus lies caudal to the ventriculus. In other ratites, the proventriculus is cranial to the ventriculus. The proventriculus of the rhea is small, while those of the kiwi, emu and cassowary are intermediate to those of the ostrich and the rhea. The ventriculus in the ostrich is separated from the proventriculus by a large opening that facilitates the surgical removal of ventricular foreign bodies via a proventriculotomy.

Ratites lack cellulase needed to digest plant fiber and therefore rely on fermentation of the fiber in the intestinal tract. This fermentation requires a slow rate of passage through the tract and an area where microbes can colonize without being swept away. ${ }^{16}$ This is where major anatomic differences exist among ratites.

Ostriches and rheas have a comparatively longer rectum and larger ceca than the emu and cassowary, perhaps indicating their development as hindgut fermenters of relatively poor-quality fodder. The ventriculus of the cassowary lacks a koilin lining. The emu and cassowary, 


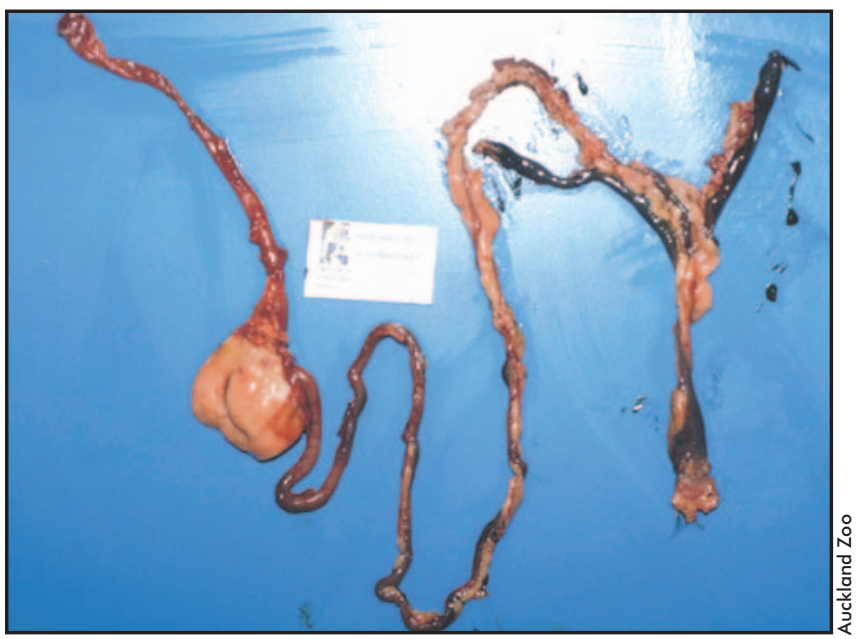

Fig 41.5 | Dissected normal gastrointestinal tract from a kiwi showing paired ceca.

Table 41.2 | Gastrointestinal

Transit Times in Ratites

Species Transit time

\begin{tabular}{|l|c|}
\hline Ostrich (immature) & 36 hours \\
\hline Ostrich (mature) & 48 hours \\
\hline Emu & $5-6$ hours \\
\hline Cassowary & - \\
\hline Rhea & - \\
\hline Kiwi & $5-20$ hours \\
\hline
\end{tabular}

evolving on a comparatively higher quality diet, have much longer small intestines, but small, non-functional ceca and a relatively shorter rectum. Overall, the intestinal tracts of the emu and cassowary are relatively shorter than those of the rhea and ostrich. The kiwi has long, paired ceca and a very short rectum (Fig 41.5). There is no gall bladder in the ostrich or emu, while the cassowary has one. Gut transit times vary with age and species (Table 41.2). For example, the emu has the capability of retaining larger fibrous particles in the ileum, allowing for greater fermentation.

\section{RESPIRATORY SYSTEM}

Despite their adaptations to a terrestrial lifestyle, ratites have retained a respiratory tract similar in structure and function to that of other avian species. Unlike other birds, however, the sternum of the ratite does not move during respiration. It is fixed to enable it to support the weight of the bird when resting. Respiration, therefore, relies on the lateral movement of the ribs, a fact that should be considered during anesthesia and recovery. ${ }^{63}$

Like other avian species, the tracheal rings of most ratites are complete cartilaginous rings. The exception is the emu, where a longitudinal cleft 6 to $8 \mathrm{~cm}$ long is found on the ventral surface of the trachea 10 to $15 \mathrm{~cm}$ cranial to the thoracic inlet. A membrane covers this cleft. As the emu matures, the cleft develops into an expandable pouch, which can be inflated to produce a booming noise in hens and a growling noise in cocks. ${ }^{31}$ This can be a complication during anesthesia if positive pressure ventilation is used, as gases may be directed into this pouch and away from the respiratory system. This can be overcome by wrapping the base of the neck with a non-adhesive bandage during anesthesia. ${ }^{31,63}$

The syrinx in ratites is poorly developed compared to other birds, a reflection on their lack of vocalization. Although the anatomy of the air sacs is similar to that of other birds, their capacity is much reduced. ${ }^{63}$

The respiratory rate varies according to age and ambient temperature. Ostrich chicks have respiratory rates of 12 to 60 breaths per minute, compared to the adult resting rate of 6 to 12 per minute. In hot conditions, this adult rate can increase to 40 to 60 breaths per minute..$^{31,34}$

\section{REPRODUCTIVE SYSTEM}

Like other birds, most ratite hens develop only the left ovary and oviduct. The exception is the kiwi, which has two functional ovaries and oviducts. ${ }^{14}$ The oviduct(s) open into the urodeum. In the ostrich, it is possible to pass a guarded culture swab into the first few centimeters of the caudal oviduct through this opening from the urodeum. This opening can be found within a small mound on the left side of the urodeum, in the 10 o'clock position. On the ventral floor of the proctodeum is a small genital mound. In the ostrich, a small $(1$ to $3 \mathrm{~cm})$ phallic-like structure arises from this mound. An even smaller structure is present in the emu, but only the mound itself is present in the cassowary and rhea.

Ratite cocks have a phallus. This differs from the mammalian penis in that there is no urethra within it, and it plays no role in urination. Ostrich and kiwi phalluses are solid structures, unlike those of the emu, cassowary and rhea, whose phalluses are spiral-shaped with a cavity and a partially inverted sleeve that everts during erection. This gives the appearance of a urethra, but it is a blind-ended structure. ${ }^{31}$ In all ratites, the non-erect phallus is found on the floor of the proctodeum. During erection, the phallus becomes engorged and is everted from the cloaca. Semen is directed along a dorsal phallic sulcus into the hen's cloaca.

The sex of ratites can usually be determined by palpation or examination of the cloaca (Table 41.3). In small chicks, the cloaca can be everted by digital pressure and visually examined. In older chicks and adults, one or more fingers can be inserted into the proctodeum and the ventral floor palpated to detect the presence or absence of a phallus (Fig 41.6). It must be noted that in 
Table 41.3 | Sex Differentiation of Ratites ${ }^{31}$

In the much smaller kiwi, cloacal palpation is difficult, and the length of the bill and tarsus (both being longer in the larger female) generally determines sex. This difference becomes evident at approximately 6 months of age.

\begin{tabular}{|c|c|c|c|c|}
\hline Species & Juvenile, Male & Juvenile, Female & Adult, Male & Adult, Female \\
\hline Ostrich & $\begin{array}{l}\text { Phallus round in cross section, } \\
1-4 \mathrm{~cm} \text { long, dorsal sulcus }\end{array}$ & $\begin{array}{l}\text { Phallic-like structure flattened in } \\
\text { cross section, } 0.5-1.0 \mathrm{~cm} \text { long, } \\
\text { no sulcus }\end{array}$ & Phallus $20-40 \mathrm{~cm}$, curved & $\begin{array}{l}\text { Phallic-like structure small, } \\
1-4 \mathrm{~cm} \text { long, straight }\end{array}$ \\
\hline Emu & $\begin{array}{l}\text { Hollow tube } 0.5-1.0 \mathrm{~cm} \text {, spirals } \\
\text { as bird gets older }\end{array}$ & $\begin{array}{l}\text { Genital mound, slight prominence } \\
\text { on genital mound }\end{array}$ & $\begin{array}{l}\text { Spiralled phallus, hollow tube, } \\
3-12 \mathrm{~cm} \text { long }\end{array}$ & $\begin{array}{l}\text { Genital mound, slight prominence } \\
\text { on genital mound }\end{array}$ \\
\hline Rhea & $\begin{array}{l}\text { Similar to emu, but more } \\
\text { elongated }\end{array}$ & $\begin{array}{l}\text { Genital mound with no } \\
\text { prominence }\end{array}$ & Similar to emu & $\begin{array}{l}\text { Genital mound with no } \\
\text { prominence }\end{array}$ \\
\hline Cassowary & $\begin{array}{l}\text { Phallus } 0.5-1.0 \mathrm{~cm} \text {, triangular in } \\
\text { cross-section }\end{array}$ & Small genital mound & $\begin{array}{l}\text { Triangular-shaped phallus pointed } \\
\text { caudally }\end{array}$ & Genital mound \\
\hline Kiwi & - & - & $\begin{array}{l}\text { Triangular-shaped phallus pointed } \\
\text { caudally }\end{array}$ & - \\
\hline
\end{tabular}

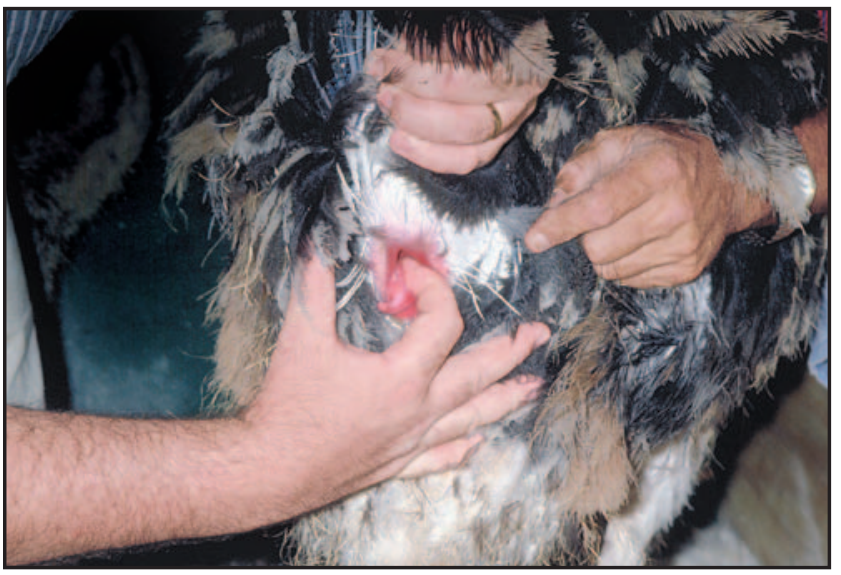

Fig 41.6 | Cloacal sexing in an ostrich to detect the presence of a phallus.

some individual juveniles, this method is inaccurate because of variations in phallic size, and the clinician must be prepared to repeat the procedure when the bird is older. Visually sexing a ratite by its size or plumage color, while often accurate, can be misleading. In the ostrich, for example, a lack of estrogen gives the cock bird its striking black plumage. However, some infertile hens have a similar lack of estrogen and have the same black plumage as cocks. These so-called "black hens" have been the subject of several lawsuits when sold as cocks without cloacal palpation to confirm sex.

The ratite cock has paired intra-abdominal testes that increase dramatically in size with the onset of the breeding season (Table 41.4). Outside of this breeding season, the testes return to their quiescent size and semen production ceases. Semen collection for assessment of fertility is unsuccessful at this time and should not be attempted. ${ }^{26}$

Ratites are seasonal breeders, although some individuals will breed year round. Ostriches, kiwis and rheas start to breed as day length increases, while emus breed as the day length starts to shorten. Cassowaries breed in late winter-early spring, coinciding with the peak availability of fruit in their natural habitat. Ostrich hens often will start
Table 41.4 | Sexual Maturity in Ratites

The age of onset of sexual maturity varies among species, sex, and differing nutritional planes and management systems.

\begin{tabular}{|l|c|}
\hline Species & Age of Sexual Maturity \\
\hline Ostrich & $24-36$ months \\
\hline Emu & $20-24$ months \\
\hline Rhea & $18-24$ months \\
\hline Cassowary & $42-48$ months \\
\hline Kiwi & $42-54$ months \\
\hline
\end{tabular}

to lay before the cock has come into peak sexual activity, so the first few eggs in each season often are infertile.

\section{URINARY SYSTEM}

Ratites have a urinary system similar to that of other birds and, like other birds, produce copious amounts of dilute urine that is resorbed and concentrated in the cloaca and caudal rectum. Ostriches are capable of expelling urine without defecating, unlike most other birds. They are unable to urinate except when standing, and an ostrich that has been recumbent for some time will have a cloaca distended with urine. ${ }^{34}$

\section{Nutrition}

In the last few years, the interest in farming some of the ratites has dramatically increased our knowledge of their nutritional requirements. However, research into this area is far from complete, and new information and concepts are becoming known each year. ${ }^{1,8,15,16,18,34,47}$ Key areas to consider in ratite nutrition are as follows:

- The adaptation to a terrestrial life-style has resulted in nutritional requirements very different from those of flighted birds.

- There are significant differences among ratites in anatomy and physiology and consequently their nutritional requirements. 


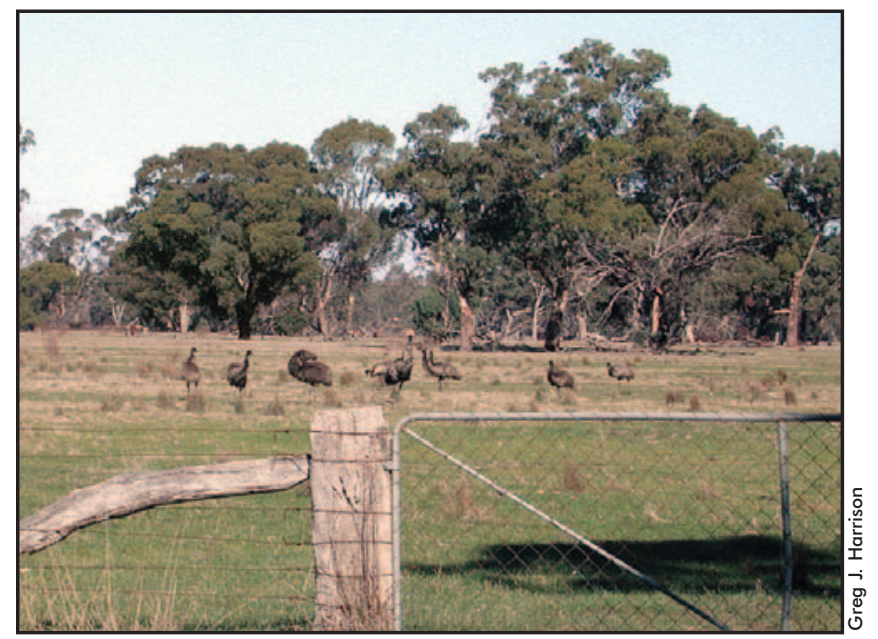

Fig 41.7 | Free-ranging emus in Australia.

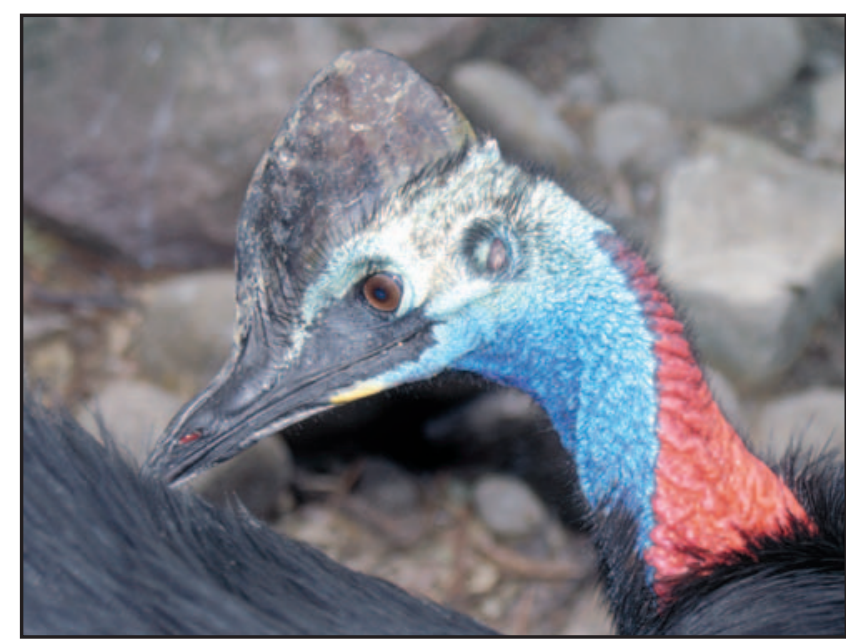

Fig 41.8 | The cassowary is primarily frugivorous.
- There are significant differences between juvenile and adult ratites (of the same species) in anatomy and physiology and consequently their nutritional requirements.

- There are economic aspects of feeding farmed ratites that must be considered when formulating diets.

The terrestrial lifestyle of ratites does not require the easily digested and rapidly utilized high-energy diets needed by flighted birds. Many ratites in their natural habitat live in semi-arid environments, grazing on bulky, high-fiber, low-quality roughage. Lacking cellulase to digest this fiber, ratites have to rely on fermentation of the fiber in their relatively long intestinal tracts to obtain the full benefit of their diet. Emus (Fig 41.7), with comparatively shorter intestinal tracts and vestigial cecum, are able to selectively retain larger fiber particles in the distal ileum allowing fermentation. ${ }^{1}$

A notable exception is the cassowary (Fig 41.8), living in rain forests and sclerophyll forests of New Guinea and North Queensland, which is primarily frugivorous (fruit eating) although other plants and even small animals are readily eaten. The kiwi feeds in leaf litter, eating 40 to $45 \%$ small invertebrates, 40 to $45 \%$ earthworms and 10 to $15 \%$ plant material. ${ }^{18}$

Juvenile ratites differ markedly from the adults. In the first week of life, the abdomen is dominated by the presence of the large yolk sac. Juvenile ratites, in their first few weeks of life, are nutritionally dependent on the quality of the parental diet and its effect on the yolk composition. The yolk provides fat, fat-soluble vitamins and some protein, and the yolk sac gradually reduces in size as the yolk is utilized. At the same time, the gastrointestinal tract increases in size. By 2 to 3 weeks of age, the yolk sac is usually completely resorbed, and the abdominal viscera are dominated by the proventriculus, cecum and large intestine. Fiber digestibility parallels this same trend. In the ostrich, for example, fiber digestibility at 3 weeks of age is only $6 \%$, increasing to $58 \%$ by 17 weeks. Fat digestibility also increases in a similar fashion, except that the 3-week-old chick has a fat digestibility of approximately $44 \% .^{1,28}$

With the development of the ratite industry as a commercial enterprise (with meat, oil and leather as its products), economic factors become pivotal in diet selection with the desire to reach a good slaughter weight at an early age. In much of South Africa, for example, ostriches are traditionally slaughtered at $\mathbf{1 4}$ months of age. This situation is rapidly changing toward younger age processing. There are some types of ostriches that can attain body weights in excess of $95 \mathrm{~kg}$ as early as 7 to 8 months of age (Black, personal communication). However, the economic benefits of being able to slaughter birds months earlier than previously attained must be offset against the disadvantages of this rapid weight gain, namely angular limb deformities and other growth abnormalities.

Ratite nutrition, therefore, requires the development of diets for each species, as well as each life stage. ${ }^{1}$

\section{PRACTICAL FEEDING}

Diets for the two main commercial species, the ostrich and emu, have been researched in some detail, but specific information on the other species is lacking. Prestarter and starter diets should be fed ad libitum (Tables $41.5,41.6)$. Older birds should be fed 1.0 to $1.5 \mathrm{~kg}$ of a formulated diet per day. As the bird matures, the amount of good-quality fiber can be increased and the amount of formulated diet reduced. At 4 to 6 months, fiber should be no more than $33 \%$ of the diet; this can be increased to up to $60 \%$ in birds weighing more than $95 \mathrm{~kg}$. The weight gains of birds on these diets, while slightly less than that of birds on $100 \%$ formulated diet, 
Table 41.5 | Diet Recommendations for Emus ${ }^{1,8,34,47}$

\begin{tabular}{|c|c|c|c|c|c|c|}
\hline Diet & $\begin{array}{c}\text { Age } \\
\text { (months) }\end{array}$ & $\begin{array}{l}\text { Est. Body } \\
\text { Weight } \\
\text { (kg) }\end{array}$ & $\begin{array}{l}\text { Crude } \\
\text { Protein } \\
\quad(\%)\end{array}$ & $\begin{array}{l}\text { Calcium } \\
(\%)\end{array}$ & $\begin{array}{l}\text { Fiber } \\
(\%)\end{array}$ & Comments \\
\hline Starter & $0-1$ & $0.5-1.3$ & $17-25$ & 1.1 & $>4$ & Feed ad lib. \\
\hline Grower & $1-16$ & $2-40$ & $16.5-23.0$ & 0.9 & $>4$ & Can be used as a maintenance diet (see below). \\
\hline Market & - & - & 14 & 0.7 & $>4$ & $\begin{array}{l}\text { Higher energy diet fed in the } 2-3 \text { months prior to slaughter in } \\
\text { order to maximize fat deposition. }\end{array}$ \\
\hline Breeder & $20-24$ & $40-45$ & $15-25$ & $\begin{array}{l}2.1 \\
\text { (Females } \\
\text { only) }\end{array}$ & $>4$ & $\begin{array}{l}\text { Feed } 1.0-1.5 \mathrm{~kg} / \mathrm{bird} \text { per day. Intake drops by } 30-50 \% \text { with } \\
\text { onset of the breeding season and increases again at the end } \\
\text { of the season. This diet is nutrient dense and is fed ad lib from } \\
1 \text { month prior to breeding till } 1 \text { month after egg laying ceases. }\end{array}$ \\
\hline Maintenance & $16+$ & $40-45$ & $12-20$ & 0.9 & * & $\begin{array}{l}\text { Intake varies with day length, peaking 1-2 months before max- } \\
\text { imum day length and then dropping as day length decreases. }\end{array}$ \\
\hline
\end{tabular}

Table 41.6 | Diet Recommendations for Ostriches ${ }^{1}$

\begin{tabular}{|l|c|c|c|c|c|}
\multicolumn{1}{|c}{ Diet } & $\begin{array}{c}\text { Age } \\
\text { (months) }\end{array}$ & $\begin{array}{c}\text { Est. Body } \\
\text { Weight } \\
(\mathrm{kg})\end{array}$ & $\begin{array}{c}\text { Crude } \\
\text { Protein } \\
(\%)\end{array}$ & $\begin{array}{c}\text { Calcium } \\
(\%)\end{array}$ & $\begin{array}{c}\text { Fiber } \\
(\%)\end{array}$ \\
\hline Pre-starter & $0-2$ & $0.8-10.5$ & 25 & $1.2-1.5$ & - \\
\hline Starter & $2-4$ & $11-28$ & 21.5 & $1.2-1.5$ & $>4$ \\
\hline Grower & $4-6$ & $29-52$ & 17 & $1.2-1.5$ & $>4$ \\
\hline Finisher & $6-10$ & $53-90$ & 13.5 & $0.9-1.0$ & 6 \\
\hline Post-finisher & $10-20$ & $91-110$ & 8.5 & $0.9-1.0$ & - \\
\hline Maintenance & Mature & - & 8 & $0.9-1.0$ & $6-60$ \\
\hline Breeder + & Laying & - & 14 & $2.0-2.5$ & $8-60$ \\
\hline
\end{tabular}

are not significantly less. This feeding regime can result in a cost savings of up to $80 \% .^{16}$

\section{Emus and Ostriches}

See Tables 41.5, 41.6 for practical feeding recommendations for ostriches and emus.

\section{Rheas}

Given the anatomic similarities of the digestive tracts of ostriches and rheas, it is reasonable to assume that their nutritional requirements are similar. ${ }^{1}$ A survey of freeranging greater rheas in Argentina showed that cultivated pasture, especially alfalfa, made up 39 to $63 \%$ of the diet. Grasses and rye made up the bulk of the balance, with seed and small invertebrates making up only a small portion. ${ }^{46}$

\section{Cassowaries}

The short length of the cassowary's intestinal tract and its subsequent rapid gastrointestinal transit time means that cassowaries require a large volume of food - up to $10 \%$ of their body weight daily. Most zoos and wildlife parks feed each bird 4 to $5 \mathrm{~kg}$ of fruit daily, including tomatoes, bananas, apples, papayas, pears, watermelon, grapes, mangoes, plums, nectarines, cherries, kiwi fruit, figs, cantaloupe, eggplant, sweet potatoes and carrots. This often is supplemented with animal protein (day-old chicks, mice, rats), vitamins and minerals. Chicks are fed a similar diet in smaller proportions. A commercial diet is available in the USA for carnivores. ${ }^{\mathrm{e}}$

\section{Kiwis}

The Auckland Zoo in New Zealand feeds its captive kiwis a diet consisting of lean ox heart, diced fruit and vegetables, yeast, wheat germ, sunflower oil, calcium carbonate and a vitamin-mineral premix. This is supplemented with earthworms and invertebrates contained in provided leaf litter. ${ }^{39}$ Captive kiwi chicks are introduced to worms and other invertebrates from 10 days of age and then rapidly convert to the artificial diet.

\section{Behavior}

An understanding of behavior in free-ranging and captive ratites is necessary to decrease stress and maximize production in both farmed birds and zoological specimens. Research has primarily focused on the ostrich.

There are four major behavioral areas of clinical interest: social behavior, feeding behavior, courtship and breeding behavior, and behavior of chicks.

\section{SOCIAL BEHAVIOR}

In their natural state and out of the breeding season, ostriches are gregarious animals, coming together in large flocks of varying ages and sexes, particularly around water holes. Within these flocks, however, there does appear to be a family social structure, with each group headed by a dominant male. During the breeding season these large flocks dissipate, with pairs and single birds being commonly seen. Free-ranging ostriches rarely associate or interact with other species, preferring to ignore them..$^{23}$

In captivity, ostriches are generally run together as pairs and trios. Interaction between other ostriches seems to be mainly confined to males pacing the fence alongside each other in a territorial display. Occasionally this behavior can be detrimental to reproductive success, as males spend more time confronting each other than they do mating. However, colony breeding of ostriches is not uncommon, and confrontation does not appear to 
be a hindrance to production.

Rheas are not nomadic birds, as they are rarely subjected to the effects of drought. One cock and several hens will reside in a family territory during the breeding season, with other cocks vigorously expelled. However, these territorial disputes disappear outside the breeding season, and flocks of up to 50 birds may form. ${ }^{40}$

Ostriches, emus and rheas in general show little aggression toward each other outside their breeding season. ${ }^{40}$

Cassowaries, on the other hand, tend to be loners, living a solitary existence within an apparently defined territory. When meeting another cassowary or another animal, cassowaries react aggressively, stretching their bodies and rumbling lowly. If this fails to dissuade the intruder, an aggressive attack may follow. For this reason, they should not be housed with other species, and humans bushwalking in their natural territory need to be cautious, particularly during the breeding season..$^{55}$

With the exception of the Stewart Island brown kiwi, where juveniles stay with their parents in a social group for at least their first year, kiwis also are highly territorial, solitary birds. ${ }^{49}$

\section{FEEDING BEHAVIOR}

Time-activity budget studies of wild ostriches indicate that they spend approximately 33\% of the daylight hours feeding. The amount of time spent feeding was affected by flock size and sex. In smaller flocks more time was spent in vigilant behaviors, watching for predators and rivals. Males spent more time being vigilant than females. As flock size increased, females spent more time feeding while males decreased their feeding time. It is thought that this may be due to increased rivalry among males in larger flocks. This behavior is replicated in captive birds. ${ }^{23}$ Captive females appear to have longer feeding bouts due to their higher energy demands associated with egg production. Males, on the other hand, feed for shorter periods due to competitiveness with other males. ${ }^{56}$

Ostriches prefer to eat natural vegetation, apparently preferring it to a formulated diet. Green annual grasses and forbs are preferred, although leaves, flowers and fruit also are consumed. Ostriches also eat stones, presumably to aid in the grinding and digestion of food. Abnormal feeding behaviors, such as the consumption of dirt and branches, are thought to be due to stress. ${ }^{23,34}$

Emus prefer high-quality foodstuffs, grazing on green plant materials, seed heads, ripening fruit, berries and insects. ${ }^{47}$ This higher quality diet may be a reflection of their shorter digestive tract and decreased gut transit times, 5 to 6 hours as opposed to 48 hours in ostrich.
Cassowaries feed predominantly on fallen fruit, but will eat almost anything, including dead rats, birds, small reptiles and fungi. As frugivores, they play an important role in the rain forest ecology, spreading undigested seeds throughout their territory. It is recorded that 21 species of rain forest plants require passage through the cassowary's digestive tract to germinate. A cock bird rearing chicks will pick up a food item and drop it in front of the chicks, clapping his beak to draw their attention to the object. ${ }^{55}$

Kiwis are unique among ratites in being strictly nocturnal and having a highly developed sense of smell. The slit-like paired nostrils are situated at the distal tip of the bill and are driven into the soil and leaf litter in search of a wide range of invertebrate prey. These include caterpillars and pupae of moths, larvae and adult beetles (especially chafer beetles), wood lice (slaters), centipedes, millipedes, slugs, snails, cicada nymphs, crickets, wetas, ants and spiders. ${ }^{49}$ Berries and seeds of native plants such as hinau (Elaeocarpus dentatus) and miro (Prumnopitys ferruginea) also form a small but significant part of the diet. ${ }^{49}$

\section{COURTSHIP AND BREEDING BEHAVIOR}

\section{Ostriches}

Ostriches breed in a defined breeding season, usually during the spring and summer months. Hens will usually display courtship behavior before the cocks and may even lay the first few eggs before being mated. The hen displays in front of an appropriate mate, lowering her head and spreading and fluttering her wings (Fig 41.9). She opens and closes her beak rapidly, making a soft noise as she does so.

Male courtship behavior begins more slowly. He develops a deep red coloration of the beak and featherless skin over the tarsometatarsal area. In some species, the skin of the neck and thighs also develops a red blush. Aggression toward other males increases, and the erect phallus is prolapsed from the cloaca and displayed. Territorial and display behavior such as booming and kantelling increase. The cock drops to his hocks, spreads his wings and swings his head from side to side, striking his back at the end of each swing.

When both birds are ready to mate, the hen will sit down and extend her head and neck along the ground. The cock approaches and sits astride her, just off to the right. The phallus enters the hen's cloaca and mating proceeds with some kantelling behavior displayed. Mating takes approximately 1 minute.

The free-ranging ostrich has a communal nesting system, 


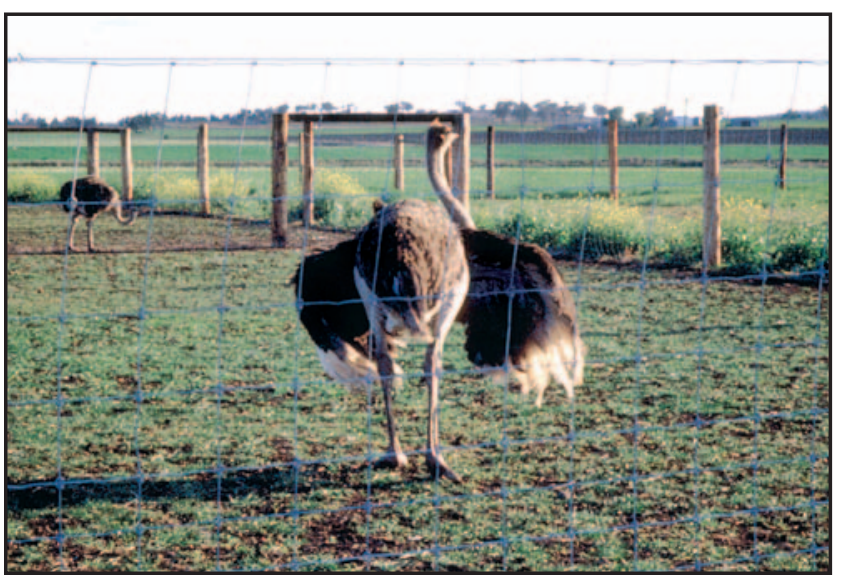

Fig 41.9 | A mature ostrich hen shows reproductive behavior by opening her wings and making a soft "cluck".

usually utilized by one cock, one "major" hen, and two "minor" hens. ${ }^{40}$ The cock digs a number of shallow scrapes, one of which is chosen by the major hen. Egg laying proceeds in this nest, but the other minor hens may lay in the same nest. The major hen lays approximately 11 eggs; the minor hens may contribute another 15 to 25 eggs. Egg laying occurs in the late afternoon and early evening, during which time both cock and hen attend the nest. The clutch size builds up over a period of up to 30 days, but incubation does not begin until the clutch is complete. Once the major hen starts to incubate, she will selectively remove many of the eggs of the minor hens from the center of the nest, pushing them to the periphery of the nest where they do not develop..$^{23}$

\section{Emus}

Emus also are seasonal breeders, laying their eggs in late autumn and winter. ${ }^{71}$ Emu hens are dominant to the cock and make a drumming noise during the breeding season. The male responds with a growling noise. Emu males are primarily monogamous, with one cock pairing with a hen for the breeding season. ${ }^{44}$ When ready to mate, the hen drops to the ground, extending her cloaca. The cock drops to his hocks behind her and intromission occurs. The cock picks the back of the hen's neck during mating. ${ }^{33}$

Emu hens start their laying by depositing eggs randomly around a pen. When a nest site is chosen, the remaining eggs are laid there and the dispersed eggs are collected by the cock and relocated to the nest site. After 6 to 10 eggs have been laid, the cock will start to incubate the eggs. Although further eggs laid near him are rolled under to join the others, in many cases the hen will stop laying or move on to find another non-incubating mate. ${ }^{59}$ At this stage the cock will hardly move, standing only to turn the eggs. His body weight will significantly decrease - a limiting effect on production. ${ }^{71}$

\section{Rheas}

Rhea cocks also incubate the eggs, but a hen may seek out more than one mate. Cocks will defend their territory and nest from other males through physical aggression such as shoving and beak grasping. ${ }^{40}$ Once the cock has established his territory, he attracts hens with his courtship displays and behavior. He prepares a shallow depression in the ground as a nest, into which multiple hens will lay. Females lay every 2 to 3 days until the nest contains approximately 13 to 30 eggs, at which point the cock will not allow the hens' access to the nest. The eggs are incubated for approximately 35 to 40 days, with the chicks hatching synchronously over 24 to 28 hours.

\section{Cassowaries}

Cassowaries breed in late winter/early spring coinciding with the peak availability of fruit. As the breeding season approaches, the hen becomes more tolerant of cocks and eventually breeding pairs form. A pair remains together for several weeks until the hen is ready to lay in a shallow scrape in the rain forest floor. The male displays to the hen by "dancing" around her, inflating his esophagus and emitting a series of low booming noises. The hen reciprocates by standing with her neck upright and her head tucked down, emitting a low rumble while vibrating her neck. When she is ready to mate, the hen sits down; the cock approaches and, if the hen does not move away, he pecks at her neck, squats and mates from behind. The whole process takes 30 minutes. ${ }^{55}$ Once the eggs are laid, the hen leaves and takes no further interest in them. She may seek out another cock and repeat the whole ritual. Up to 8 eggs may be laid in a single clutch, although it is rare that this many hatch and survive. ${ }^{55}$

\section{Kiwis}

Kiwis live in stable pairs within a territory that may be aggressively protected. They lay only two eggs, one from each ovary, usually 24 days apart. ${ }^{14}$ They are usually laid in a burrow and are incubated by the male. (The exception is the Stewart Island brown kiwi, where the female does assist incubating the eggs, possibly due to the colder weather). Eggs are laid between July and February, and lost clutches are not immediately replaced. The male emerges from the burrow each night for a few hours to feed until within a few days of the eggs' hatching. During the incubation period (70-90 days), the male can lose up to $17 \%$ of his body weight. ${ }^{52}$

\section{BEHAVIOR OF CHICKS}

In the natural state, hatching of ostrich eggs takes place over 2 to 3 days, during which time the chicks remain "brooded" by an adult. When all have hatched, the chicks form crèches of up to 30 individuals, overseen by 


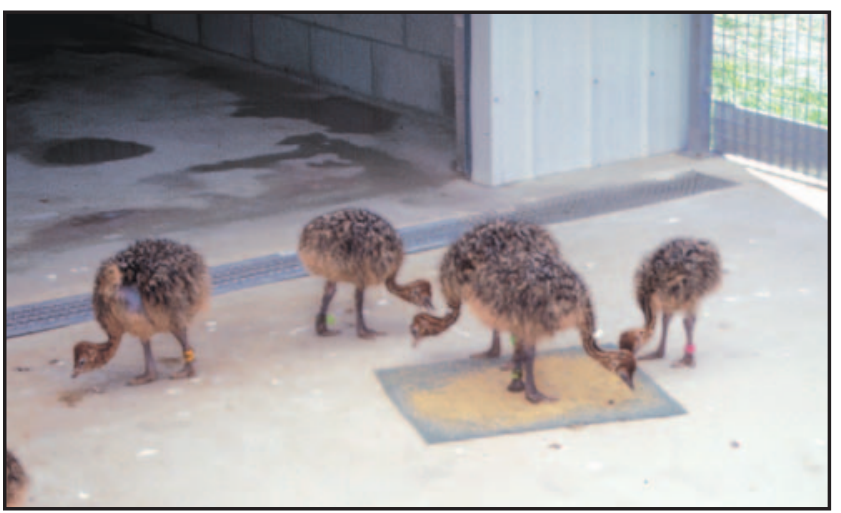

Fig 41.10 | Ostrich chicks feeding. Note the colored mat, which attracts the chicks' attention.

a single pair of adults. These crèches often will merge when they meet, although older chicks are not usually accepted into younger groups. When the chicks are approximately 1 year old, they are left by their guardians in compact peer groups to fend for themselves. ${ }^{23}$

Ostrich chicks are naturally gregarious and do not do well if reared in solitude. ${ }^{23}$ Lacking parental security and the comfort of their crèche, isolated chicks (both freeranging and captive-bred) will suffer severe stress and anxiety. ${ }^{34}$ Captive-bred chicks imprint on their human caretakers and look to them for parental security. If the caretaker leaves, the chicks become stressed, leading to decreased food intake, gastric stasis and eventual death. This can be overcome by setting a routine for the chicks in their first 6 weeks (better enabling them to deal with stressful situations), and by having a caretaker with the chicks as much as possible. ${ }^{42}$

A time/activity budget study of captive ostrich chick behavior showed that chicks aged 7 to 14 days spent equivalent amounts of time feeding from the floor and walking around their enclosure $(27.7 \%$ and $23.1 \%$, respectively). ${ }^{23}$ Lacking an adequate thermoregulatory capacity, chicks must spend some time under a brooder lamp warming themselves (11.2\%). Presumably, freeranging chicks would fulfill this requirement by brooding under an adult guardian. The balance of their day was spent pecking at objects (10.2\%), standing (6.7\%), drinking (5.8\%) and feeding from a tray (3.5\%).

This study would indicate that feeding chicks from a tray or dish is less successful than placing the food directly on the ground (Fig 41.10). As 10\% of their time was spent pecking at other objects, it would appear that pecking is a means for ostriches to locate food. As they learn by mimicry, this behavior spreads rapidly through a group of chicks. Another study indicated that chicks prefer the colors green and white, pecking at these colors in preference to other colors. ${ }^{24}$
Aberrant behaviors, such as pecking at non-food items and other chicks, can become highly repetitive and habitual. Pecking at other chicks may be an aggressive behavior but is more likely to be misdirected feeding behavior or a response to stress. Once this behavior becomes habitual, it is difficult to stop. Chicks that peck the most have the slowest growth rates and may even die. ${ }^{23}$

\section{Emus}

Emu chicks spend their first week primarily huddled under a heat source. Any activity is done as a group and is usually initiated by older chicks. Feeding as a group is of short duration; as an individual, it is even shorter. As the chicks get older, they become more active, spending more time running, chasing, pecking at objects and feeding. Sudden disturbances will induce clustering behavior, all the chicks coming together in a huddle. Emu chicks, like ostriches, learn by mimicry, usually by watching the behavior of older chicks. ${ }^{30}$

\section{Rheas}

Rheas also rear their chicks in crèches, overseen by a single male. Newly hatched chicks are readily adopted into these crèches with good survivability of the new chicks. Smaller chicks adopted into groups of larger chicks have a higher mortality. ${ }^{41}$ The precocial chicks, gray with darker stripes, will stay with the male for approximately 6 months, often taking shelter under the wings of the male when he lies down. Juvenile birds may stay together for several years until reaching sexual maturity (Smith, personal communication).

\section{Cassowaries}

Cassowary cocks look after their chicks until they are about 9 months old, although chicks of 16 months have been seen accompanying the cock. When the chicks are approaching maturity, the cock chases them away and they establish their own territories. ${ }^{55}$

\section{Kiwis}

The kiwi hen, while not sharing the incubation with the cock, remains nearby and spends time with the male and chicks once they are hatched. Chicks are precocial and independent within approximately 14 to 20 days, but remain with or near their parents for up to several years. With the exception of the Stewart Island brown kiwi, kiwi chicks are fully independent after their first 3 weeks of life. ${ }^{49}$

\section{Production Management}

With the advent of commercial farming of some ratites species and improved captive breeding of others in 
zoological parks, the management of these birds has received much attention in recent years. Decreased production and most disease problems in ratites often can be traced directly to management faults and errors. An understanding of the management principles of ratite production is, therefore, essential when assessing problems with these birds.

With the modernization of the ostrich industry, more research on management of this species has been done than for any other ratite. Unless otherwise stated, the following information refers to ostriches. Care must be taken in extrapolating this to other ratites.

\section{EGGS}

\section{Incubation}

Artificial incubation of eggs is widely practiced in all ratite species in an endeavor to increase production. Egg characteristics and incubation requirements for ratite species are contained in Table 41.7.

Given healthy, fertile eggs, there are nine parameters for successful artificial incubation: selection of eggs for incubation, egg collection and sanitation, egg storage, incubator temperature, relative humidity, ventilation, turning and positioning of eggs, hygiene and monitoring and record keeping.

\section{Selection of Eggs for Incubation}

In the early days of the recent ostrich "boom," every egg was worth a lot of money, and eggs that should not have been incubated were set. The lesson learned was that these eggs rarely hatched, or, if they did, they produced weak chicks. Not only was the labor intensity in maintaining these eggs and chicks not comparable to the results obtained, but they also acted as "disease multipliers", adding greatly to farm problems.

Undersized eggs or those with poor shell quality have a tendency to lose excessive moisture content during incubation, producing weak, dehydrated chicks. Conversely, oversized eggs or those with excessively thick shells do not lose enough moisture, and the resultant chicks are edematous and weak. Neither type of chick survives well.

It is therefore not advisable to set eggs that are heavily contaminated, cracked, undersized (eg, ostrich eggs less than $900 \mathrm{~g}$ ), oversized (eg, ostrich eggs greater than $1500 \mathrm{~g}$ ) or that have poor shell quality (eg, too thick or too thin).

\section{Egg Collection and Sanitation}

Microbial contamination of eggs can be responsible for significant losses of fertile eggs, with infection rates
Table 41.7 | Egg Characteristics and Incubation

Requirements of Ratite Species ${ }^{62,66}$

\begin{tabular}{l|c|c|c|c|c|}
\hline \multicolumn{2}{|c}{ Ostrich } & Emu & Rhea & Cassowary & Kiwi \\
\hline $\begin{array}{l}\text { Eggs per } \\
\text { year }\end{array}$ & $40-60$ & $20-40$ & $40-60$ & $3-10$ & $1-6$ \\
\hline $\begin{array}{l}\text { Egg weight } \\
\text { (g) }\end{array}$ & $900-1700$ & $500-700$ & $400-700$ & $500-700$ & $400-450$ \\
\hline $\begin{array}{l}\text { Incubation } \\
\text { period (days) }\end{array}$ & $41-43$ & $48-50$ & $36-41$ & $47-54$ & $70-90$ \\
\hline $\begin{array}{l}\text { Temperature } \\
\left({ }^{\circ} \text { C) }\right.\end{array}$ & $36.0-36.4$ & $35.2-35.5$ & $36.0-37.2$ & $36.1-36.7$ & $35.5-36.5$ \\
\hline $\begin{array}{l}\text { Relative } \\
\text { humidity (\%) }\end{array}$ & $22-36$ & $35-50$ & $55-70$ & $55-70$ & $60-65$ \\
\hline
\end{tabular}

ranging from 13.4 to $67 \%$ of dead-in-shell ostrich eggs. Most isolates are of fecal or soil origin. ${ }^{22}$ Clearly, success rates can be improved if this contamination is minimized or avoided.

Ratites should be provided with clean, dry, well-drained nest sites. Many birds will accept an artificial nest site, so care should be taken to locate it in an easily accessible area that meets the previous requirements. Wherever possible, eggs should be collected several times daily, with the last collection just before sunset. This regime will minimize the contact time the egg has with possible contaminants, which may be drawn through the shell as the egg cools.

Sanitation of eggs remains controversial, but obvious gross contamination should be removed with a soft, dry brush. The decision on whether to then wash the eggs, fumigate them or irradiate them with ultraviolet (UV) light will depend on local experience and available equipment. One study indicated that UV irradiation gave better hatchability results than washing but was only marginally better than doing nothing at all. ${ }^{65}$ In this study, washing the eggs in a disinfectant bath resulted in decreased hatchability. If washing is required for badly contaminated eggs, the wash solution should be maintained at $40^{\circ} \mathrm{C}$ to minimize the solution being drawn through the eggshell pores.

\section{Egg Storage}

The realities of modern farming practices mean that ostrich eggs should be set only once or twice weekly. This allows a better allocation of labor and other resources. As embryonic development does not appear to proceed at temperatures under $25^{\circ} \mathrm{C},{ }^{25}$ it is feasible to store eggs below this temperature for periods not exceeding 7 days without significant effects on hatchability. A common practice is to store the eggs at 15 to $20^{\circ}$ $\mathrm{C}$, turning them twice daily for 3 to 7 days. As the storage time increases, early embryonic mortality rises and can reach $100 \%$ by 17 days. ${ }^{22}$

Prewarming the eggs prior to storage may increase 
hatchability. It has been demonstrated that heating to $36^{\circ} \mathrm{C}$ for 4 hours prior to less than 6 days' storage at $17^{\circ}$ $\mathrm{C}$ increased hatchability by $8 \% .{ }^{11}$ The same study showed that warming stored eggs for 16 hours prior to incubation had no significant effect on hatchability.

\section{Incubator Temperature}

Incubation temperatures below those recommended in Table 41.7 may result in slow-developing chicks that hatch late; conversely, temperatures that are too high may lead to premature hatching.

The recommended temperatures may represent a compromise between the higher temperatures required for an early embryo and the lower temperature needed for a late embryo generating its own metabolic heat. ${ }^{22}$ Most ratite producers compensate for this metabolic heat by moving late-term embryos to a hatcher operating at $1^{\circ}$ to $2^{\circ} \mathrm{C}$ lower than the incubator. These hatchers also operate at a higher relative humidity, and turning is stopped.

\section{Relative Humidity}

As the embryo develops, the egg loses moisture (and, therefore, weight) through the shell. Numerous studies have shown that hatchability is maximized when this weight loss is between 12 and 18\%. The amount of moisture loss is dependent on the porosity of the shell and the relative humidity of the air surrounding the egg. As most incubators can only increase the relative humidity of room air (they lack the ability to dehumidify it), it is recommended that they be placed in an air-conditioned room fitted with dehumidifiers.

Most ratite eggs incubated at the recommended relative humidity shown in Table 41.7 will lose 12 to $18 \%$ of their weight during incubation. However, variables such as shell porosity, altitude, room relative humidity and incubator characteristics can all affect the degree of weight loss. Regular weighing of eggs allows for adjustment of the relative humidity in order to achieve the desired weight loss.

\section{Ventilation}

The embryonic cells utilize oxygen, and carbon dioxide is produced. The embryo exchanges these gases initially across the yolk sac membrane and later across the chorioallantoic membrane. Without an efficient exchange, the embryo will die. The rate of exchange is determined by the porosity of the shell and the gas concentration gradient across the shell. It is, therefore, imperative that the incubator maintain a constant flow of fresh air over the eggs, usually achieved by a fan. ${ }^{25}$ Kiwis are an exception and are best incubated in a still-air incubator. At Auckland Zoo, kiwi eggs are cooled outside the incubator for 1 hour per day during the first 55 days of incuba- tion. ${ }^{66}$ This simulates egg temperatures recorded in the nest corresponding to the departure of the male for foraging during natural incubation. ${ }^{19}$

\section{Turning and Positioning of Eggs}

Failure to turn eggs results in poor development of extraembryonic membranes, a reduction in the amount of extra-embryonic fluid produced and poor utilization of albumen by the embryo. Turning is, therefore, essential during incubation up to transfer to a hatcher. ${ }^{22,25}$ Various degrees and rates of turning have been described, but hatchability increases as frequency increases up to 8 times daily. Beyond this, there is little difference in hatchability. The degree of turning is dependent on the frequency of turning, with low-frequency turning requiring large-degree turns. ${ }^{22}$

Incubating eggs horizontally for 2 weeks and then vertically for the remainder of incubation appears to maximize ostrich egg hatchability. ${ }^{22}$

Kiwis turn their large eggs up to 180 degrees in the early stages of incubation but, due to the imbalance caused by the large air cell, can only rock them from side to side in the later stages. ${ }^{19}$ Artificially incubated eggs are turned on a 2-day cycle, 45 degrees at a time. On the first day, the egg is turned clockwise 90 degrees in two stages and then 90 degrees counterclockwise in two stages. On the second day this is reversed. No turning is done after 55 days' incubation. ${ }^{66}$

\section{Hygiene}

The buildup of microbial contamination can lead to embryonic and chick deaths through bacterial infection. It is, therefore, essential that strict hygiene measures be in place in the incubator room and that the incubator is regularly cleaned and fumigated. Bacterial colony counts can be used as a means of monitoring the level of hygiene in an incubator. ${ }^{25}$

\section{Monitoring and Record Keeping}

Regular assessment of incubating eggs by weighing and candling is essential to detect problems before they become overwhelming. Several advantages of efficient candling practices have been described, and include the early identification and removal of contaminated/ infected eggs, early identification of embryonic death at different stages, and the identification of malpositioned eggs. ${ }^{2}$ The ability to make these judgments early allows the early removal of non-viable eggs (thus freeing up valuable incubator space), minimizes contamination and assists in the identification of incubator problems.

Accurate records of setting dates, weight loss, expected hatch dates, outcomes, stages of embryonic death and 

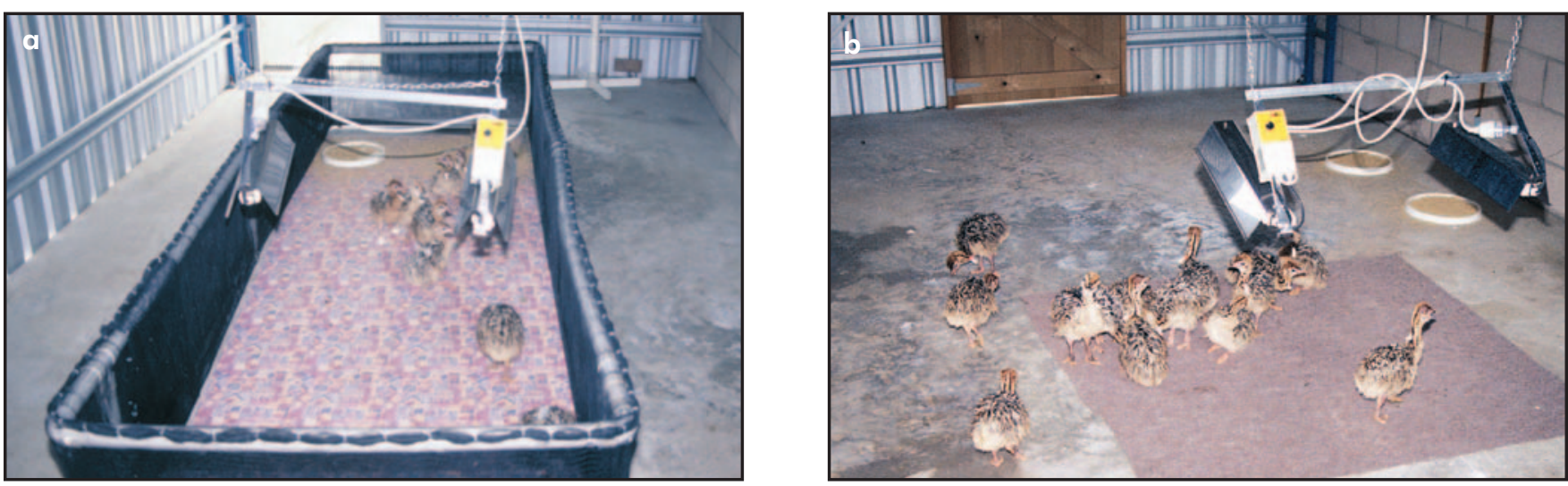

Figs $41.11 \mathbf{a}, \mathbf{b}$ | Brooder shed for ostrich chicks allows sufficient room for exercise. Note the heat lamp.

subsequent chick viability are essential for the clinician trying to assess an incubator problem. Without these records, the task is almost impossible, and much time will be lost monitoring production and generating new records. Producers must have the necessity for good record-keeping strongly impressed on them.

\section{CHICKS}

Incubation techniques for ratite eggs have improved to a point where it is reasonable to expect 70 to $90 \%$ of fertile eggs to hatch. Adult ratites are generally robust animals with few serious health problems. Chick survivability is, therefore, the greatest limitation to successful production. Reported mortality rates of chicks less than 3 months of age range from 10 to $50 \%$, decreasing to $10 \%$ between 3 and 6 months, and 5\% up to 12 months. ${ }^{68}$

The ratite chick is precocial, ie, independent of its parents, but its early viability is dependent on the input of the hen into the egg. Calcium for bone development and growth initially comes from the eggshell; minerals, protein and vitamins come from the albumen; fat and immunoglobulins come from the yolk. Therefore, parental nutrition is a key factor in chick survivability. Other factors, such as egg size, weight loss during incubation, microbial contamination and genetics also will have an effect.

Ostrich chicks in particular are susceptible to the effects of stress. Stressors such as temperature extremes, inadequate ventilation, overcrowding, inadequate exercise, poor hygiene, social stresses and incorrect nutrition are all detrimental to growth and survivability of chicks. ${ }^{28}$ Management of chicks requires that these stressors and others are identified and minimized (Figs $41.11 a, b$ ).

While the yolk sac and its contents are important to the chick, the theory that chicks should not be fed for the first few days to encourage absorption of the yolk appears to be incorrect. With the notable exception of kiwis, ratite chicks need to start eating as early as possi- ble to allow the functional development of the gastrointestinal tract. ${ }^{68}$ They may need an older chick or a chicken to teach them to eat, and the feeding behavior of chicks should be utilized to encourage them to eat as soon as possible. Kiwi chicks begin to probe for food after 5 to 7 days, but rarely begin feeding until their yolks have been fully absorbed at approximately 10 days of age (JakobHoff, personal communication). Because the nutrition of ratite chicks is significantly different from that of adults, dietary management is essential to achieve optimal growth rates. Growth rates of chicks as well as the incidence of problems related to nutrition (such as angular limb deformities) should be monitored.

Many different chick-rearing systems have been used around the world. Concrete runs, sand, bare ground, mobile sheds, permanent structures, lucerne (alfalfa) pasture have all been utilized under different conditions. The one constant seems to be that what works well in one geographical area does not work nearly as well in other areas. Variables such as genetics, climate, available nutrition and labor cost all have an effect on the suitability of chick-rearing systems.

A unifying concept in farmed ratite chick rearing is that of "all in-all out" - the batching of chicks according to their age and body weight and the maintenance of these groups as they progress through the farm system. The advantages of this system have been described and include tailoring the rearing system to the age of the group, moving the birds less so there is less stress, and minimizing the spread of disease by preventing chick movement in or out of the group. ${ }^{68} \mathrm{~A}$ review showed that "mixed weight" groups grew more slowly than "same weight" groups, indicating that social pressures affected the growth of chicks. ${ }^{24}$ Such social pressures may be less pronounced in groups of chicks of similar ages and weights.

Management of chicks must aim to minimize stress and maximize growth rates. Biosecurity, vermin control, farm 
traffic flow, quarantine and isolation of sick chicks also are necessary to reduce losses due to disease. The integration of these two concepts is paramount to the success of chick rearing.

\section{BREEDERS}

Ostriches appear to be induced breeders, ie, the hen requires the stimulation of the presence of a male in order to lay eggs. This makes tools such as artificial insemination, commonly used in the poultry industry, less useful and more complicated. ${ }^{17}$ The use of vasectomized "teaser" birds may be one option, but practicality and economics would require fertile cocks to run with egg-laying hens. This can be done as pairs, trios or colonies.

Colonies of ostriches can range in size from 9 to 150 birds, with males making up 20 to $30 \%$ of the flock. Although colony breeding is recommended by some authors ${ }^{33}$ on the grounds of economics, fertility and ease of management, others believe that pairs and trios are more productive. ${ }^{23,34}$ Additionally, selection of the best breeding stock requires accurate records of breeding success and chick performance/data that cannot be obtained from colony breeding.

The separation of sexes out of the breeding season and their reintroduction at the start of the season can be a powerful stimulus for breeding. ${ }^{33}$ This management tool often is overlooked or neglected, usually due to space constraints.

\section{Emus}

Emus are primarily monogamous and will pair with only one hen per season. After mating, the cock broods the eggs and takes no further interest in breeding. In this species, artificial insemination may be advantageous. ${ }^{44}$ Many Australian farms allow breeding birds to run as a flock and then separate pairs as they form. This allows compatibility to develop and retains some degree of genetic selection.

\section{Cassowaries}

Cassowaries, by nature solitary and potentially aggressive, may not tolerate the presence of a mate outside the breeding season. This needs to be assessed on a case-bycase basis. Young birds paired early appear to make the best breeders, as adults newly introduced to each other may take several years to develop a pair bond. As the breeding season approaches, cassowaries should be placed within visual proximity to each other, separated by a fence. When they are seen to be interacting, they can share the same enclosure. ${ }^{55}$

\section{Kiwis}

Captive kiwis must be paired carefully. These territorial birds are likely to fight when first introduced and can inflict fatal wounds with their strong feet and claws. Generally, new pairs are established by placing them in adjoining pens so they can hear and smell each other for some weeks to months before an introduction is attempted. Introducing the birds outside the breeding season in a neutral territory and with widely separated feeding stations and roosting boxes can minimize fighting. However, even with these precautions it is important that the introduction and first few nights together be closely supervised, either by video or direct observation. Once the birds have settled down and are eating regularly, a successful pairing can be assumed. Kiwis tend to pair for life, but have been known to form a new pair bond should one of the birds die (Jakob-Hoff, personal communication).

\section{FLOCK HEALTH SCHEMES}

With the advent of the commercial phase in ostrich and emu farming, ie, the production of meat and leather as distinct from breeder (investment) birds, the emphasis of veterinary services has moved away from the individual bird to the overall production of the farm. Veterinary input now focuses on areas such as farm design, general management, selection of stock, nutrition, and disease control and prevention. ${ }^{27}$ An indication of the effect of various parameters on farm profitability is shown in Table 41.8.

These figures are based on production of a farm in South Africa. The magnitude and ranking of these parameters will vary slightly from farm to farm and from country to country. Obviously, some of these, especially the income per slaughter bird, chick mortality rates, hen productivity and feed cost, can have a major impact on profitability. These parameters are well within the capacity of veterinarians to have an effect through a farm flock health scheme. A well-designed and carefully implemented ostrich flock health scheme is a feasible way of achieving improved performance and profitability. ${ }^{7}$

Before implementing a scheme, the veterinarian first needs to assess the following:

- The farm layout and facility design

- The management practices employed in all aspects of the farm from incubation to breeder management

- The nutritional basis of all rations currently in use

- Complete performance records over the last 2 to 3 years

- Any major problems encountered - past or present

- The health status and quality of the ostriches of all ages 
Table 41.8 | Effects on Farm Profitability ${ }^{7}$

\begin{tabular}{|l|c|c|}
\hline Parameter & Increase by & Effect on profitability \\
\hline Income per slaughter bird & $10 \%$ & $55 \%$ increase \\
\hline Chick mortality & $10 \%$ & $35 \%$ decrease \\
\hline Eggs/hen and hatchability & $10 \%$ & $31 \%$ increase \\
\hline Feed cost & $10 \%$ & $27 \%$ decrease \\
\hline Cost of contract work & $10 \%$ & $10 \%$ decrease \\
\hline Labor cost & $10 \%$ & $3 \%$ decrease \\
\hline Cost of drugs & $10 \%$ & $1 \%$ decrease \\
\hline
\end{tabular}

From this, a written plan can be prepared along the following lines:

- Evaluation of the present layout and performance of the farm

- Issues requiring improvement and their relative priority

- Realistic targets for future performance

- Aspects of a flock health scheme required to attain such targets

- Requirements for ongoing monitoring

This plan must take into consideration the farmer's willingness and ability to participate in such a scheme, his/her aims and goals, and the economic realities of implementing the scheme. The scheme must be costeffective for the farmer or it will not succeed. ${ }^{27}$

Effective implementation of flock health schemes involving facility design, record keeping, genetic selection programs, preventive health measures, quarantine principles, biosecurity practices, nutritional analysis and monitoring of management practices can improve the efficiency of ostrich farms and increase financial returns to the ostrich producer. ${ }^{7}$

\section{Clinical Examination}

\section{DISTANT EXAMINATION AND HISTORY}

As with any animal, the examination of a ratite begins with a distant examination and history taking. As most ratites being examined will be farmed birds or zoological specimens, a history of their management, diet, previous medical history and behavior is vital in understanding the problem presented to the clinician.

While taking a history, the clinician is well advised to stand back and watch the bird before capture and restraint takes place. Species' differences in behavior must be understood. For example, it is normal for a cassowary to be isolated and aloof. However, an ostrich that is doing the same may well be ill. Chicks behave differently than adults. Abnormalities in gait, respiration, appetite, posture or feathering often can be best assessed before the bird is stressed by capture. A fecal examination should be conducted if possible. When as much information as possible has been collected, the bird should be restrained in order to be more closely examined.

\section{HANDLING AND RESTRAINT}

Not only are many of the larger ratites capable of inflicting serious injury on people, but also their economic value often lies with the condition of their hide and meat. As such, handling and restraint techniques must ensure that no harm befalls either the bird or the people doing the handling. Time spent planning and preparing to work with a ratite is never wasted!

Wherever possible, adult ostriches should be removed from their own enclosure (which they have come to regard as their territory) before an attempt is made to handle them. If time permits, allowing the birds to become accustomed to being fed in a smaller enclosure (eg, a shed or mobile cattle yard) for a few days can make capture simple and safe. Once the birds are comfortable entering the enclosure, merely closing a gate behind them can capture them. If time does not allow this approach, several people working together can herd the birds into a corner of the pen or into a small enclosure. As ostriches are quite adept at running past individual handlers, creating a "mobile fence" by suspending a length of shade cloth between handlers can effectively herd the birds to where they can be captured. This whole procedure must be done calmly and smoothly, with a minimum of running, shouting and arm-waving.

Once confined, individual ostriches can be caught and examined. An ostrich is generally more amenable when it is "hooded", ie, a tubular cloth hood is placed over its head restricting its eyesight. To do this, the neck is grasped just below the head, either by hand or with a shepherd's crook, and the head is pulled down to a horizontal plane (Fig 41.12). Care must be taken not to damage the neck and jugular vein, as fatal hematomas have resulted from trauma to the vein by careless handling. The handler has the hood bunched up over the free hand, which then grasps the beak (Fig 41.13). The hood is then pulled over the head and the head is released. The ostrich is then immediately restrained with a hand on the cranial sternum and another on the pubic bones. Large birds may require two handlers, one on either side of the bird. Under no circumstances should the bird be given the opportunity to run while hooded, as injury invariably results.

\section{Emus}

Emus do not tolerate a hood, although Jakob-Hoff (personal communication) has found them useful as long as 


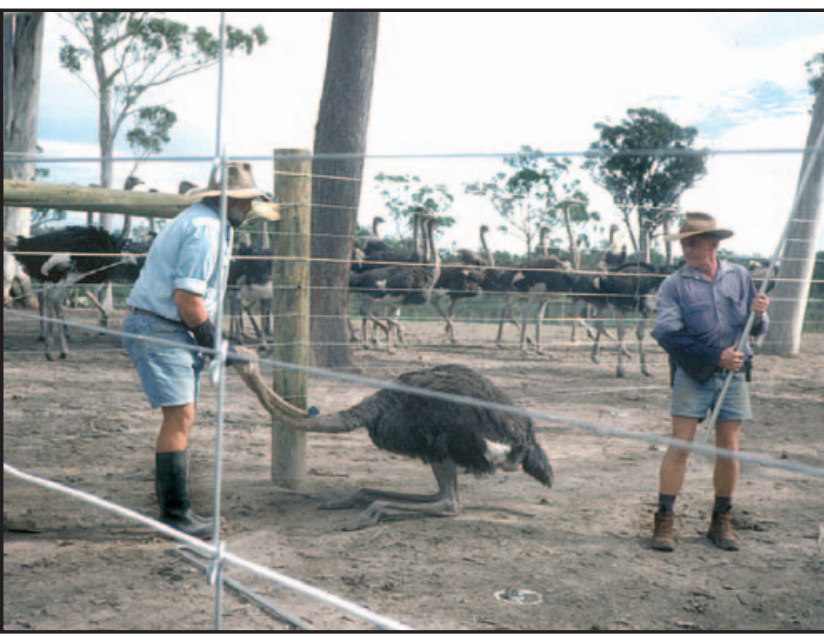

Fig 41.12 | Capture and restraint of an ostrich using a "shepherd's crook" and a hood.

the hooded bird, can detect no light. Once placed in a small enclosure, approaching an emu from behind and hooking an arm around the base of the neck near the thoracic inlet can restrain it. The bird is pulled back against the handler's body and the other hand is used to grasp the upper neck. The bird is then tilted backward into a more human-related upright position relative to the normal emu body position. Once it has settled, the upper neck can be released. ${ }^{45}$

\section{Cassowaries}

Cassowaries are dangerous to handle. Physical restraint can be achieved by herding the bird into a corner. The handlers use padded sheets of plywood to block the bird. They must be braced against the impact of a running or jumping bird, and the handlers must be aware of the cassowary's ability to jump. Once the bird is cornered, it can be approached from behind and pushed firmly to the ground by applying body weight squarely onto its back. Once it is sitting, a second person can restrain the tarsometatarsus to immobilize the bird. Care must be taken not to injure the leg. A hood can be tried, but not all birds will tolerate it. ${ }^{55}$ Chemical sedation may be required for further examination. This can be given after physical restraint or by darting before examination. Ketamine, diazepam, xylazine and zolazepam-tiletamine have all been used with unpredictable results. Medetomidine was trialed at 0.26 to $0.31 \mathrm{mg} / \mathrm{kg}$ (captive birds) and 0.38 to $0.54 \mathrm{mg} / \mathrm{kg}$ (free-ranging birds) with good results. ${ }^{70}$ External stimuli must be avoided once the birds are sedated to avoid auditory stimulation.

\section{Kiwis}

Kiwis are easily restrained by grasping both legs above the hock joint between the thumb and middle finger of the right hand with the index finger between the two legs (Fig 41.14). The left hand supports the ventral and

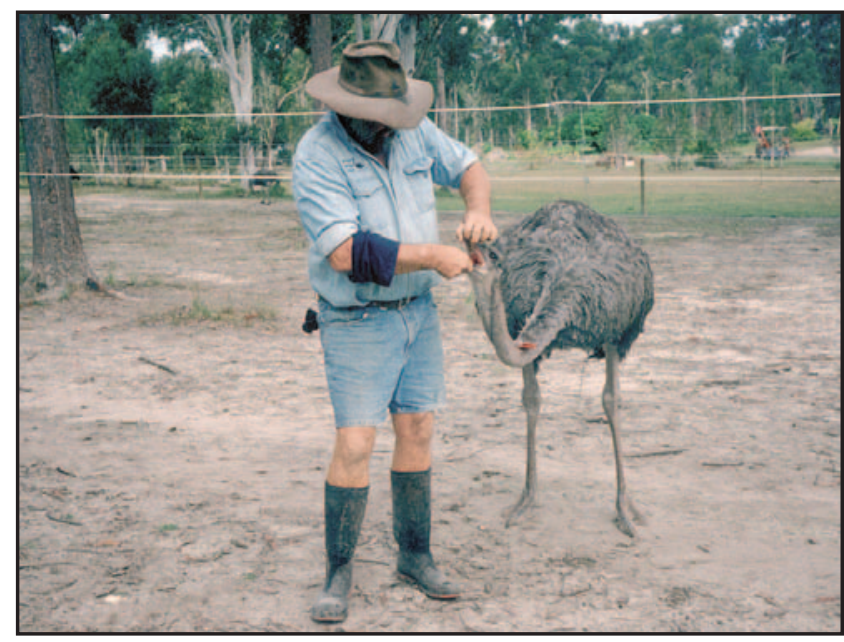

Fig 41.13 | Preparing to place a hood on an ostrich. lateral body into a sitting posture in the crook of the right elbow. Directing the bird's bill and eyes under the cover of the left arm while in this position will help calm it. An alternative method, used when handling kiwi in the field, is to grasp the two legs as described and hold the bird in the upside-down position. The dorsal body is then placed on the lap of the holder with the head similarly placed under the left arm. The birds tolerate this remarkably well for the considerable period of time it takes to apply a radio transmitter and take measurements and diagnostic samples (Jakob-Hoff, personal communication).

\section{PHYSICAL EXAMINATION}

Once the bird has been safely restrained, the physical examination can proceed (Table 41.9). A systematic and thorough evaluation is essential, and the use of a comprehensive examination form can be invaluable. A detailed guide to the examination of the ostrich is available. ${ }^{4} \mathrm{~A}$ summary of this technique can be applied to all ratites. After the bird is caught and identified (leg band, microchip), the following are performed:

- Externally examine the eyes, beak, nares, ears and oral cavity.

- Check the body condition by palpating the spine.

- Auscultate the thorax, determining the heart and respiratory rates and checking for abnormal respiratory and cardiac sounds at several sites.

- Auscultate the proventriculus and ventriculus.

- Palpate the abdomen, including the proventriculus and ventriculus.

- Observe the feet and legs for abnormalities.

- Palpate the limbs and determine the alignment of the tibia.

- Examine the skin and feathers for abnormalities and parasites.

- Examine the vent and digitally sex the bird. 


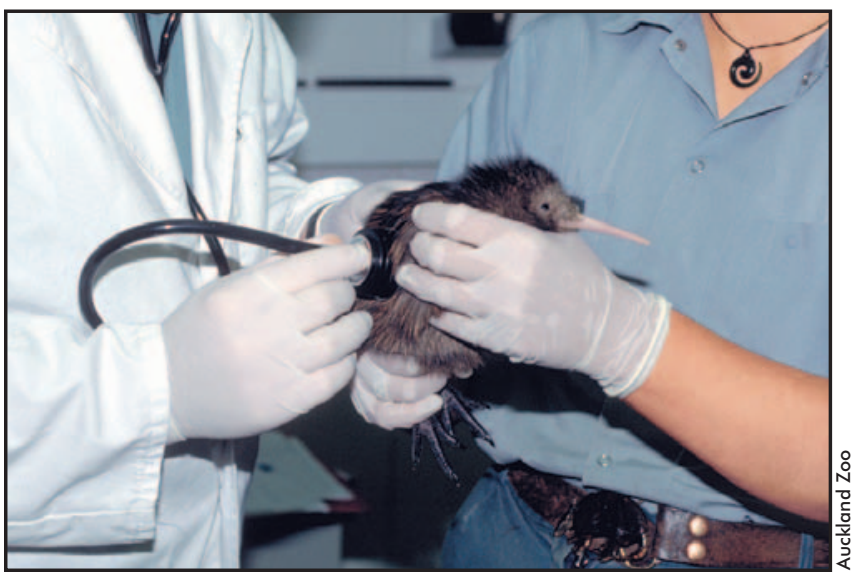

Fig 41.14 | Examining a kiwi chick at Auckland Zoo.

Table 41.9 | Some Physical Characteristics of Adult Ratites

\begin{tabular}{|l|c|c|c|}
\hline & \multicolumn{1}{c}{ Weight $(\mathrm{kg})$} & \multicolumn{1}{c|}{ Heart Rate } & Respiratory Rate \\
\hline Ostrich & $80-150$ & $30-60$ & $6-12$ \\
\hline Emu & $35-55$ & $42-76$ & $13-21$ \\
\hline Cassowary & 85 & $35-90^{*}$ & $20-44$ \\
\hline Rhea & 25 & - & - \\
\hline Kiwi & $1.5-4.0$ & $70-240$ & $12-60$ \\
\hline
\end{tabular}

*Recorded under sedation

Clinical findings should be recorded as the examination proceeds, especially if there are several birds to be examined at the same time. A standardized examination form is invaluable in this respect; not only does it allow accurate recording, it also ensures the clinician does not overlook part of the examination (see Chapter 6 Maximizing Information from the Physical Examination).

\section{DIAGNOSTIC TESTING}

If appropriate, samples for laboratory analysis can be collected following the examination.

Several veins are readily accessible for venipuncture in ratites other than the kiwi. The right jugular vein is suitable for the smaller ratites and juveniles. Care should be taken in the larger ratites, because the thin-walled jugular can tear easily, leading to a potentially fatal hematoma. The basilic vein can be used in ostriches (Fig 41.15), but the small vestigial wings in other ratites make this vein unsuitable in other species. The medial metatarsal vein is accessible in all species, but care must be taken that adequate restraint is in place before this vein is utilized. In kiwis, this is the only usable vein (Fig 41.16), the jugular being inaccessible due to the overlying layer of thick, fatty skin (Jakob-Hoff, personal communication).

A blood smear should be made immediately and the rest of the sample placed in plain tubes or, preferably, lithium heparin. If the samples cannot be analyzed rapidly, it is advisable to centrifuge the sample and refrigerate the plasma or serum.

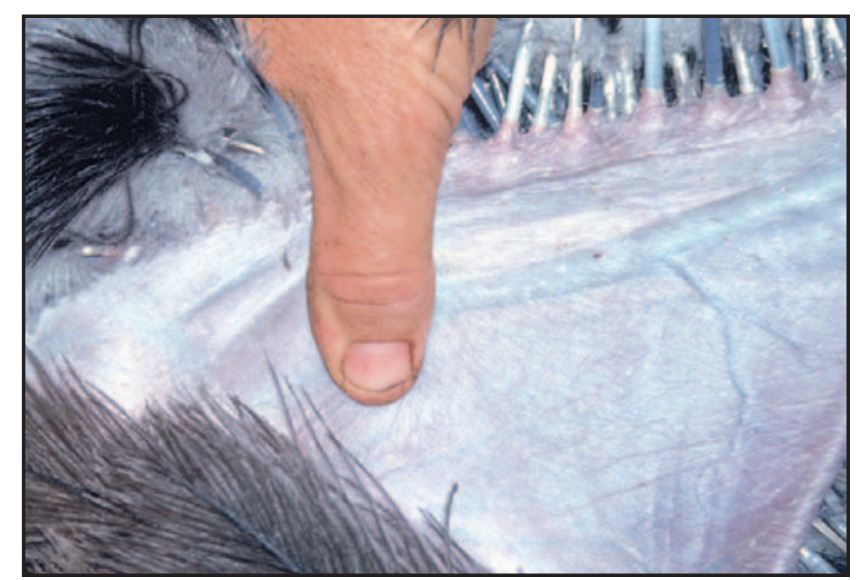

Fig 41.15 | The basilic vein is suitable for blood collection only in the ostrich.

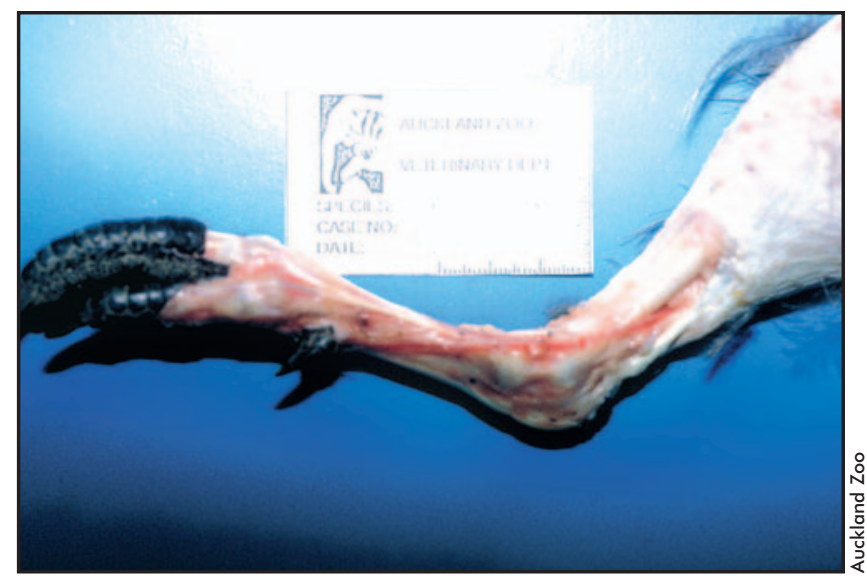

Fig 41.16 | Kiwi dissection shows the medial metatarsal vein, the only accessible vein for blood collection in this species.

In ostrich hens with a history of reproductive disease (embryonic deaths, poor shell quality, irregular laying, misshapen eggs), a swab can be taken of the distal oviduct for cytology and culture. With the hen hooded and perhaps restrained, a gloved and lubricated hand is placed into the urodeum, and the oviduct opening is located. This opening is found in the 10 o'clock position in a small papilla. A finger can be passed through this opening into the distal oviduct and a guarded mare swab introduced alongside the finger. A limitation of this technique is that only the caudal 10 to $15 \mathrm{~cm}$ of the oviduct can be accessed; a disease process occurring more proximally may be missed. Common pathogens isolated include E. coli, Pseudomonas spp. and Klebsiella spp. Fecal contamination is not uncommon. ${ }^{4}$

Several authors have described semen collection in ostriches. ${ }^{4,26,36}$ It should be attempted only in mature, reproductively active birds; young or sexually inactive birds will produce little or no semen. Although artificial insemination techniques are being developed in ostriches, semen collection and assessment are important parts of an infertility assessment. Minimal restraint should be 
Table 41.10 | Ratite Hematology and Biochemistry Reference Ranges

\begin{tabular}{|c|c|c|c|c|c|}
\hline & Ostrich & Emu & Cassowary & Rhea & Kiwi \\
\hline PCV (\%) & $45(41-57)$ & $47.4(39-57)$ & $48.1(33.5-58)$ & $45.5(29-59)$ & $46(38-54)$ \\
\hline $\mathrm{Hb}(\mathrm{g} / \mathrm{L})$ & $140-172$ & $136-170$ & $174(135-200)$ & $126(64-170)$ & - \\
\hline $\mathrm{MCHC}(\mathrm{g} / \mathrm{L})$ & $347-412$ & $352-433$ & $352-433$ & 451 (444-457) & $250(110-333)$ \\
\hline WBC (x10\%/L) & $18.7(10-24)$ & $14.9(8-21)$ & $17.55(8.6-31.6)$ & $11.8(4.1-25.7)$ & $11.6(8.7-14.5)$ \\
\hline Heterophils $\left(x 10^{9} / \mathrm{L}\right)$ & $10.8-16.6$ & $8.0-13.1$ & $11.1(6.4-20.9)$ & $7.4(0.5-20.0)$ & $6(4.0-8.2)$ \\
\hline Lymphocytes $\left(\times 10^{9} / \mathrm{L}\right)$ & $2.2-7.7$ & $1.5-6.6$ & $5(2.0-9.5)$ & $3.6(0.5-7.0)$ & $4.2(2.5-5.9)$ \\
\hline Eosinophils (x10\%/L) & $0-0.37$ & $0-0.9$ & $0.3(0.2-0.4)$ & $0.4(0.05-0.7)$ & $0.18(0.7-0.29)$ \\
\hline Monocytes (x10\%/L) & $0-0.75$ & $0-0.15$ & $1.1(0.1-2.8)$ & $0.5(0.04-1.6)$ & $0.3(0.1-0.5)$ \\
\hline Basophils $\left(x 10^{9} / \mathrm{L}\right)$ & $0-0.37$ & 0.15 & $0.4(0.19-0.8)$ & $0.4(0.07-1.6)$ & $0.56(0.09-1.3)$ \\
\hline CK (U/L) & $800-6508$ & $70-818$ & $365-1335$ & $0-2640$ & $521-971$ \\
\hline AST (U/L) & $226-547$ & $80-380$ & $269-1399$ & $20-192$ & $64-138$ \\
\hline Bile Acids $(\mu \mathrm{mol} / \mathrm{L})$ & $2-34$ & $2-30$ & - & - & - \\
\hline Serum Protein $(\mathrm{g} / \mathrm{L})$ & $24-53$ & $34-44$ & $45-75$ & $34-62$ & $54-62$ \\
\hline Uric Acid (mmol/L) & $0.59-8.9$ & $0.59-8.3$ & $0.24-4.5$ & $0.17-1.4$ & $0.3-0.38$ \\
\hline $\mathrm{Ca}(\mathrm{mmol} / \mathrm{L})$ & $2.0-3.4$ & $2.2-3.2$ & $2.3-3.0$ & $2.6-8.2$ & $1.85-3.1$ \\
\hline Glucose (mmol/L) & $9.1-18.3$ & $5.6-13.5$ & $5.5-12.8$ & $2.1-8.8$ & $3.0-3.9$ \\
\hline LDH (U/L) & $408-1236$ & $318-1243$ & - & $269-1640$ & 2380 \\
\hline
\end{tabular}

used, as excessive stress may inhibit ejaculation. The administration of 5 to 10 IU of oxytocin intravenously may produce a better sample. ${ }^{60}$ The phallus is manually extruded from the cloaca, and the dorsal base (where the ductus deferens papillae are located under the uroproctodeal fold) is gently but firmly massaged. Ejaculation occurs after a variable time period, and the semen can be collected as it runs down the dorsal phallic groove.

The use of an artificial cloaca to collect semen in emus has been described. ${ }^{44}$ Using a "teaser" hen, the artificial cloaca is placed over the phallus as the cock attempts to mate with the hen. This technique has been quite successful in collecting from these birds.

\section{Interpretation of Clinical Pathology}

The interpretation of hematology and biochemistry in ratites is very similar to that of other avian species. It should be noted that ratites normally have much higher creatine kinase $(\mathrm{CK})$ values than other avian species. Table 41.10 shows reference ranges for ratite species. An excellent guide to the interpretation of ratite clinical pathology also is available. ${ }^{32}$

\section{NECROPSY TECHNIQUES}

As the ratite industries become more commercial, the value of the necropsy to quickly and accurately determine a diagnosis and etiology continues to grow (Fig $41.17)$. Necropsy offers both the most accurate diagnosis and the most cost-effective option. These advantages should be impressed on both farmers and zookeepers, and the value of having both fail-to-hatch eggs and dead birds submitted for veterinary examination emphasized.

\section{Egg Necropsy}

All eggs that fail to hatch should be necropsied. Not only does an egg necropsy allow a determination of the stage and possible cause of embryonic death, it also provides the clinician (and farmer) with a true indication of fertility. On several occasions, this author has been asked to investigate infertility problems, only to discover (via egg necropsy) that the problem was actually one involving embryonic mortality.

The technique is simple. The egg is first weighed and, if possible, the weight loss during incubation is calculated. The shell quality is examined (surface texture, porosity and cleanliness). The egg is then candled. Candling allows identification of the air cell, an indication of its size and mobility, and an idea as to whether an embryo or infection is present. Hairline cracks in the shell also can be identified. This information is recorded and then the next step is taken.

The egg is placed vertically onto a support (a ring of PVC pipe is sufficient) with the air cell uppermost. The shell over the air cell is broken with a drill or a sharp tap of a hammer, revealing the air cell. The shell is then broken away to the level of the inner shell membrane forming the base of the air cell. This membrane is peeled away with fine forceps, revealing the egg contents (Fig 41.18a).

At this stage, a swab for culture of bacteria and fungi can be taken from the albumen or inside the shell. If an embryo is not obviously present, the blastodisk on the yolk should be examined. The yolk around this disk is less dense than the rest of the yolk, so it will float uppermost. In an infertile egg, the disc will appear as a small white point. A very early embryo will appear as a small white "doughnut" with a patch of yolk in the middle. Any blood vessel development at all is confirmation of fertility (Fig 41.18b). 


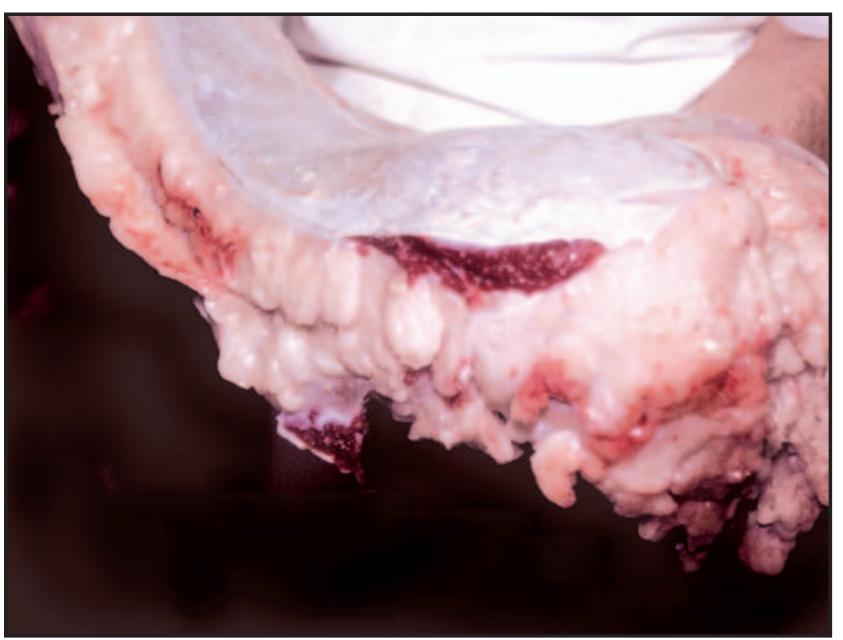

Fig 41.17 | This intra-abdominal fat in a mature ostrich at slaughter represents a significant financial loss to the farmer in wasted food conversion.

If an embryo is present, two determinations must be made: the positioning of the embryo (if it is late term) and the stage of development.

Embryonic position is observed before removing the embryo from the egg. The normal late-term embryo is positioned with the head next to the air cell, right side uppermost and turned to the right, with the beak adjacent to the right foot and shoulder; legs flexed on either side of the body and ventral to the shoulders; and the spine following the long axis of the egg. Any other position is classified as a malposition, and may account for embryonic death. Descriptions and possible causes of classical malpositions are described in Table 41.11.

A measurement of the embryo's crown-to-rump length and an assessment of its growth and development can give an indication as to whether this is an early, midterm or late-term embryo.

A procedure for egg necropsies has been described. ${ }^{12}$ In summary, once the embryo is removed from the egg, the following procedure is recommended:

1. Record position of yolk sac and percentage that is external.

2. Weigh embryo and record crown-to-rump length.

3. Measure thickness of edema (if present) over proximal neck and thighs.

4. Remove and weigh yolk sac (if necessary, incise abdomen).

5. Record gender.

6. Collect samples from the chorioallantois, yolk and proventriculus for culture.

7. Collect tissues from multiple organs, including the membranes, in formalin.

By determining the stage and cause of embryonic death, the clinician can then start to narrow down the search for
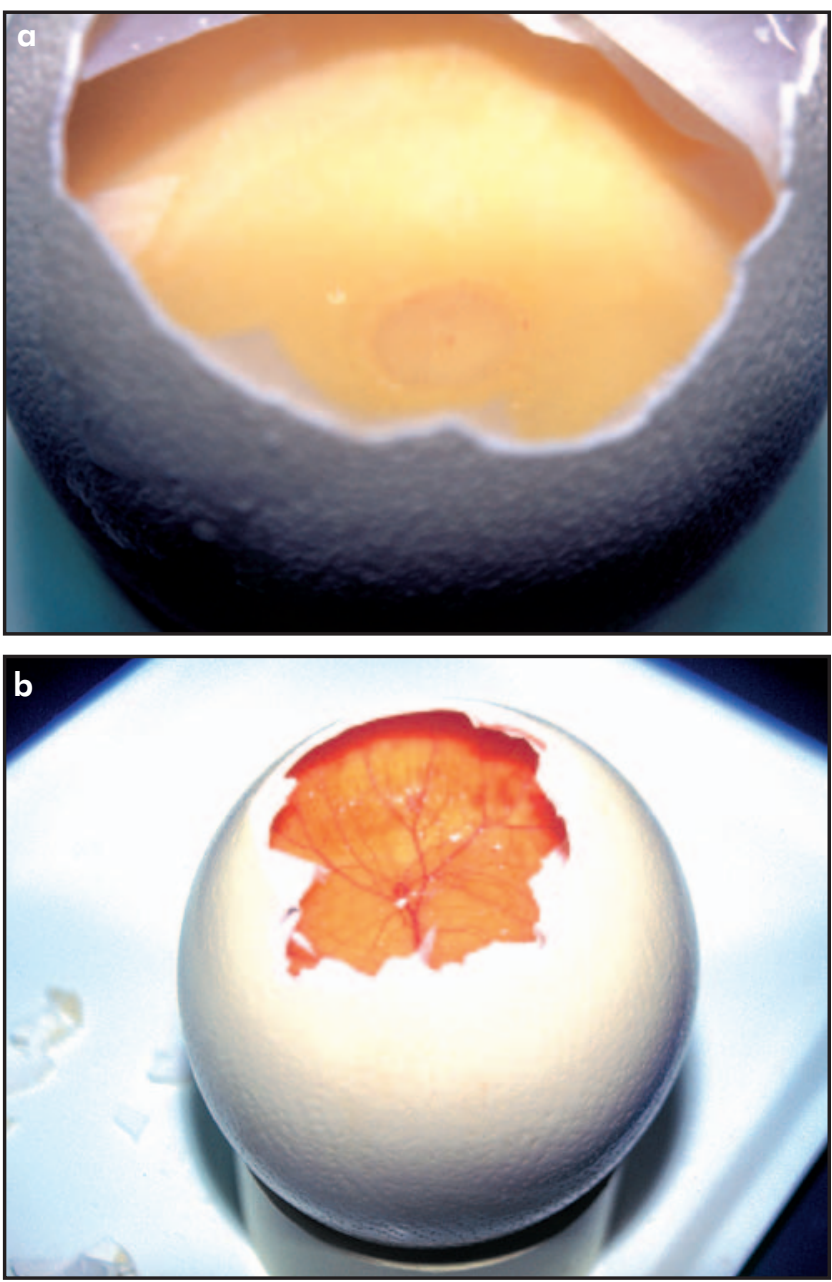

Figs 41.18a,b | An egg necropsy can identify early embryonic deaths in fertile eggs: a) degenerated germinal disc; b) degenerating vessels.

the underlying cause of embryonic mortality (Table 41.12).

\section{Necropsy of Chicks and Older Birds}

As with any necropsy, a thorough and methodical procedure is essential to obtain the best results. If the bird is still alive, it should be weighed (if possible) and blood collected for hematology and biochemistry. A physical examination should be conducted and then the bird humanely euthanized. Care must be taken to ensure that the euthanasia method does not affect likely necropsy findings.

The body can be opened through a midline incision with the bird in dorsal recumbency, but an alternative is a lateral approach. The body is placed in lateral recumbency, the upper leg is abducted, and an incision is made along the ventrolateral border of the body. The skin is reflected, the coxofemoral joint is disarticulated and the leg is further abducted. The abdominal muscles and ribs can then be transected and reflected dorsally. This gives a good exposure of the internal organs. 
Table 41.11 | Embryonic Malpositions

\begin{tabular}{|l|l|l|}
\hline \multicolumn{2}{|l|}{ Malposition } & \multicolumn{2}{l|}{ Description } & $\begin{array}{l}\text { Possible Causes (if known) } \\
\text { I I }\end{array}$ & $\begin{array}{l}\text { Head is down between } \\
\text { the legs }\end{array}$ & $\begin{array}{l}\text { Chick is rotated within the } \\
\text { egg, with the head at the } \\
\text { end opposite to the air cell }\end{array}$ & $\begin{array}{l}\text { Egg position and low } \\
\text { temperature during } \\
\text { incubation }\end{array}$ \\
\hline II & $\begin{array}{l}\text { Head is rotated to the left, } \\
\text { with the head under the } \\
\text { left wing }\end{array}$ & $\begin{array}{l}\text { Egg position, temperature } \\
\text { and parental malnutrition }\end{array}$ \\
\hline III & $\begin{array}{l}\text { Beak is away from the air } \\
\text { cell, rest of the body is } \\
\text { normally positioned }\end{array}$ & Egg position \\
\hline V & Feet over the head & \\
\hline VI & Head over the right wing & Parental nutrition \\
\hline
\end{tabular}

The relative size and position of the organs is noted, and culture swabs are taken from the liver and proventriculus. Individual organs are removed and examined, and tissue samples are placed in formalin for histological evaluation. Proventricular contents should be examined and the contents assessed for possible impaction. Intestinal contents should be assessed as to consistency and volume. A written report should be prepared and placed in the farm's records.

\section{Diseases of Ratite Chicks}

Studies of ostrich chick mortality indicate that most deaths occur before 2 months of age and that chicks of this age are most likely to die of infectious diseases. After 2 months of age, death is usually the result of leg problems. Mortality rates from all causes drop significantly after 4 months (More, personal communication). In South Africa, chick mortality is reported to be as high as 40 to $50 \%$ up to 3 months and 10 to $30 \%$ from 3 to 6 months. Mortality from 3 to 12 months dropped to $5 \% .68$ Problems with chicks are a major limiting factor in successful ratite production.

\section{DISEASES OF THE INTEGUMENT}

Congenital deformities of the beak are occasionally seen in ostrich chicks. The more common of these deformities include scissor beak and a downward deviation of both rhinotheca and gnathotheca. These rarely cause any significant problems to the chick, and treatment is rarely warranted. Severe deformities can interfere with the prehension of food and, unless the chick is valuable, euthanasia may be the preferred option. Manganese deficiency in parental diets is reported to cause a shortening of the lower beak. ${ }^{34}$

Mycotic dermatoses involving Aspergillus spp., Trichophyton spp. and Microsporum spp. have been reported. ${ }^{34}$ Treatment with $10 \%$ enilconazole wash ${ }^{a}$ is the recommended treatment.
Table 41.12 | Troubleshooting Embryonic Mortality ${ }^{40,64}$

\begin{tabular}{|c|c|}
\hline Problem & Possible Causes \\
\hline Floating air cell & $\begin{array}{l}\text { - Rough handling of egg } \\
\text { - Parental nutritional deficiencies } \\
\text { - Genetics }\end{array}$ \\
\hline $\begin{array}{l}\text { Early } \\
\text { embryonic } \\
\text { mortality }\end{array}$ & $\begin{array}{l}\text { - Parental nutritional deficiencies } \\
\text { - Delayed egg collection } \\
\text { - Incorrect storage - temperature incorrect, stored } \\
\text { too long } \\
\text { - Shaking/jarring of eggs } \\
\text { - Incubator problem - temperature, turning, } \\
\text { ventilation } \\
\text { - Formaldehyde fumigation of eggs in first } 3 \text { days }\end{array}$ \\
\hline $\begin{array}{l}\text { Mid-term } \\
\text { embryonic } \\
\text { mortality }\end{array}$ & $\begin{array}{l}\text { - Parental nutritional deficiencies } \\
\text { - Infection } \\
\text { - Inadequate turning } \\
\text { - Incubator problem - temperature, turning, } \\
\text { ventilation } \\
\text { - Lethal genes }\end{array}$ \\
\hline $\begin{array}{l}\text { Late-term } \\
\text { embryonic } \\
\text { mortality }\end{array}$ & $\begin{array}{l}\text { - Malpositions } \\
\text { - Incubator problem - temperature, humidity, } \\
\text { turning, ventilation } \\
\text { - Infection } \\
\text { - Parental nutritional deficiencies }\end{array}$ \\
\hline $\begin{array}{l}\text { Air cell pip, } \\
\text { fail to hatch }\end{array}$ & $\begin{array}{l}\text { - Hatcher problem - temperature, humidity, } \\
\text { ventilation }\end{array}$ \\
\hline Malformed chicks & $\begin{array}{l}\text { - Incubator temperature too high } \\
\text { - Parental nutritional deficiencies } \\
\text { - Genetics } \\
\text { - Teratogens }\end{array}$ \\
\hline Edematous chicks & $\begin{array}{l}\text { - Humidity too high } \\
\text { - Shell too thick } \\
\text { - Inadequate ventilation }\end{array}$ \\
\hline Sticky chicks & $\begin{array}{l}\text { - Humidity too high } \\
\text { - Shell too thin }\end{array}$ \\
\hline External yolk sac & $\begin{array}{l}\text { - Temperature too high } \\
\text { - Edematous chick } \\
\text { - Inappropriate intervention to assist hatch }\end{array}$ \\
\hline
\end{tabular}

Avipoxvirus has been reported in ostrich chicks in South Africa, Israel, the United States and Australia.. ${ }^{53,58}$ The proximity of poultry and the presence of suitable vectors contribute to outbreaks. Chicks from the age of 2 weeks are affected, with both cutaneous and diphtheritic forms reported. Vaccination from 10 days of age with fowl pox vaccine combined with biting insect control will usually limit or prevent outbreaks.

Hyperkeratosis of the skin around the head, eyelids, beak commissures, neck and feet has been observed in ostrich chicks and is thought to be nutritional in origin. Supplementation with zinc and biotin often is curative. It must be distinguished from mycotic infections.

A biotin-responsive plumage abnormality has been recorded in wild-hatched North Island brown kiwi chicks simultaneously parasitized by a hemoparasite of the genus Babesia. The feather abnormalities included complete feather loss from the ventral-lateral trunk and thighs and retention of keratinized sheaths on dorsal contour feathers, giving the plumage a dry, spiky texture. The hemoparasite infection was associated with pronounced pyrexia, and it was speculated this might have interfered with biotin absorption as has been recorded in poultry. ${ }^{38}$ 


\section{DISEASES OF THE GASTROINTESTINAL TRACT}

Candidiasis in ratite chicks can result from immunosuppression, malnutrition or prolonged antibiotic therapy. Plaque-like lesions can be observed in the oropharynx, although the infection can extend through to the ventriculus. Cytology and/or culture are diagnostic. Treatment with nystatin or ketoconazole is usually successful.

Other fungal infections, including zygomycosis and macrorhabdosis (megabacteriosis), have been reported in ostrich chicks. ${ }^{34,58}$ Treatment is the same as in other avian species.

Bacterial enteritis is a common problem in ostrich chicks (Fig 41.19 ). Salmonella spp., E. coli and Pseudomonas spp. are the most common isolates. Clinical signs are not limited to just the intestinal tract, as septicemia frequently develops. Diarrhea, swollen joints, keratitis, corneal ulceration, depression and anorexia are common clinical signs. Hepatic granulomas are frequently seen on necropsy of chicks dying from Pseudomonas septicemia or colibacillosis. Although antibiotic therapy, based on culture and sensitivity testing, can save some chicks, severely affected birds usually die. It is essential that investigating clinicians do all that is possible to identify the source of the infection, as management issues significantly contribute to such outbreaks.

Clostridial enteritis may be an over-diagnosed problem. Clostridium perfringens is a normal inhabitant of the ostrich intestinal tract and its identification on fecal culture does not necessarily constitute a diagnosis of clostridial enteritis. ${ }^{34}$ Abrupt dietary changes, starvation, stress, anthelmintics and vaccinations have been associated with outbreaks. Ingestion of soil and substrate contaminated with clostridial spores also may be a contributing factor. Acute mortality, occasionally with antemortem anorexia and depression, is the hallmark of this infection. Necropsy reveals varying degrees of enteritis associated with clostridial spores, and Clostridium spp. can be cultured from affected chicks. Treatment with tetracycline, penicillin and zinc bacitracin has been described. ${ }^{34,58}$ A vaccination protocol using $\mathrm{Cl}$. perfringens Type B and D vaccines at either 1 week or 1 month, or at 3 and 6 weeks, also has been described. ${ }^{34}$

Adenoviral enteritis has been described in ostrich chicks in the USA. ${ }^{54}$ Affected chicks, usually older than 2 months, show marked depression, anorexia and diarrhea. Mortality is usually greater than $90 \%$. Vertical and horizontal transmission can occur. Infectious bursal disease has been reported in the United States. Affected chicks show depression, anorexia and diarrhea over a 3to 4 -day period before death. ${ }^{58}$

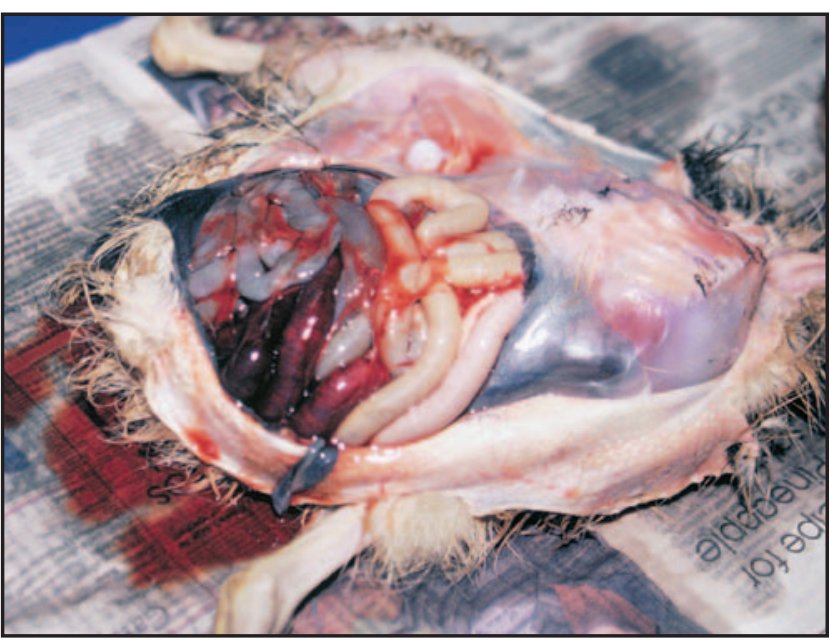

Fig 41.19 | Intestinal volvulus in a 6-week ostrich chick. This may be secondary to enteritis and is usually diagnosed postmortem.

Endoparasitism can be a major problem in young ratites, particularly if they are reared with adults or have access to adult fecal material. An excellent review of ratite parasites is available. ${ }^{21}$ Libyostrongylus douglassii (wire worm) is a major problem on some ostrich farms on most continents, with mortality rates of affected chicks approaching 50\%. It causes a diphtheritic proventriculitis (vrotmaag: rotten stomach), with clinical signs including weight loss, depression, anorexia and death. Strongyle eggs identified on fecal flotation should be cultured, as larval identification is needed to diagnose L. douglassii. Levamisole, fenbendazole and ivermectin have been reported as effective treatments.

Many other helminths have been identified in ostriches and other ratites, including trichostrongyles, ascarids, acanthocephalans and cestodes. Protozoal parasites, including Cryptosporidia, Balantidium spp., Histomonas meleagridis, Spironucleus and coccidia, have been reported in many ratite species. Coccidiosis is by far the most important gastrointestinal parasite of captive kiwis, and regular monitoring is important. Cumulative fecal samples collected over 4 days are examined to reduce the possibility of missing this intermittently shed parasite. The infestation is readily treated with a single dose of toltrazuril ${ }^{\mathrm{b}}(25 \mathrm{mg} / \mathrm{kg} \mathrm{PO}) .{ }^{37}$

Prolapses of the cloaca and rectum in young chicks can occur secondarily to enteritis. Treatment of these prolapses involves replacement with a gloved, lubricated finger and correction of the initiating cause. If the prolapse recurs, the use of a purse-string suture may be required. This suture can be placed under local anesthesia and should be left in place for no more than 3 days (Black, personal communication).

Treatment of enteritis in ostrich chicks revolves around 
several key principles: early recognition that a problem exists; identification and isolation of affected chicks; identification of the causative pathogen; and appropriate treatment.

Batching of chicks and good biosecurity measures offer the best chance of success in stopping an outbreak.

\section{DISEASES OF THE RESPIRATORY SYSTEM}

The artificial environment in which young ratites are usually reared - hatchers, brooders and chick pens often are poorly ventilated, and respiratory disease results.

Aspergillosis is a common disease in ratites (Fig 41.20). Aspergillus fumigatus and A. flavus are commonly isolated from affected birds; A. niger and A. terreus may not be clinically significant (Black, personal communication). In chicks, the disease is associated with poorly ventilated hatchers and brooders. Eggs contaminated with Aspergillus spp. from nest substrate placed in a hatcher cause a buildup of spores, which are then inhaled by newly hatched chicks. Alternatively, chicks placed in poorly ventilated, unhygienic brooder pens may be exposed to spores. If an immunocompromised chick inhales sufficient spores, an overwhelming fulminating "brooder pneumonia" may result, often followed by relatively acute death. In other cases, an air sacculitis may result, which may not become clinically apparent for weeks, months or even years. Treatment is usually unrewarding because of the often chronic, granulomatous nature of the disease. Ketoconazole, amphotericin $\mathrm{B}$ and itraconazole have been used to treat systemic aspergillosis, while inhalation of smoke generated by enilconazole bombs ${ }^{c}$ have been used to treat pneumonia and air sacculitis.

\section{Pseudomonas aeruginosa, Pasteurella haemolytica, $P$.} multocida, Haemophilus spp. and Bordatella spp. have been isolated from chicks with respiratory infections. ${ }^{34}$ Mycoplasma spp. also has been isolated.

An important factor in the pathogenesis of respiratory disease in ratite chicks is ammonia toxicosis. Ammonia arising from the feces and urine builds up in chick sheds overnight when the chicks are closely confined. Overcrowding and poor ventilation in the shed will lead to ammonia toxicosis. This should be suspected when, upon opening the shed in the morning, the chicks are lethargic and yawn frequently. In more severe cases, keratitis may develop. The smell of ammonia in the shed is strong. The irritation to the airways can lead to secondary infections as described above. Prevention revolves around management: overcrowding should be avoided

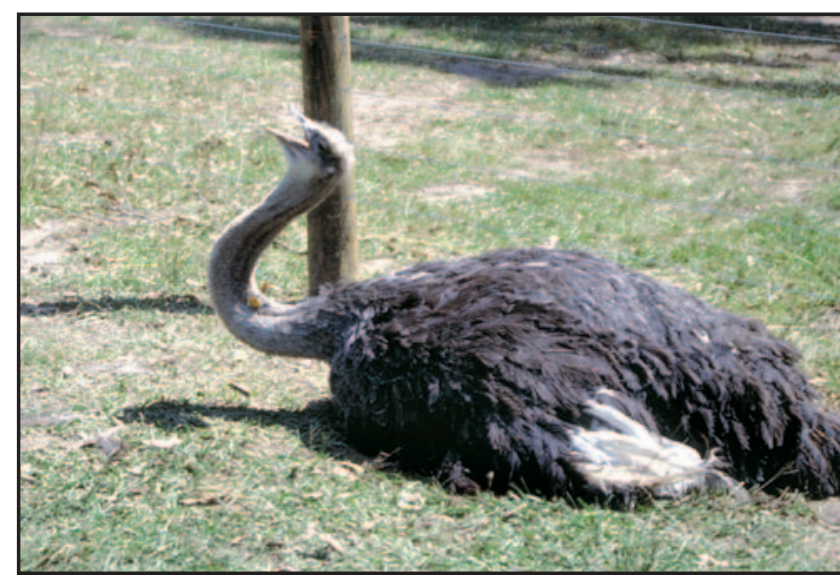

Fig 41.20 | An ostrich hen with advanced aspergillosis exhibits severe respiratory distress.

(allow 0.5 to $1.5 \mathrm{~m}^{2}$ floor space per chick). Chick sheds should be cleaned daily to reduce the buildup of feces and urine, and ventilation should be improved. As ammonia is heavier than air and will therefore settle closer to the floor, ventilation must be provided at chick level. Ventilation grills 15 to $30 \mathrm{~cm}$ above the floor combined with exhaust vents in the roof can dramatically improve the situation.

\section{DISEASES OF THE YOLK SAC}

The newly hatched chick receives some nutrients and possibly immunoglobulins from the yolk sac, which is attached to the intestine via the vitello-intestinal duct. Yolk sac contents are drained by this duct and via absorption by mesenteric blood vessels around the yolk sac. A few days before hatching, the yolk sac is drawn into the abdomen, and the abdominal muscles close over it at the navel. The yolk is resorbed over 10 to 14 days, and only a vestigial remnant remains after 3 weeks.

Occasionally the umbilicus fails to close fully, exposing a portion of the yolk sac. If the exposure is only small, dressing it with iodine and bandaging until closure is complete may be all that is required. Larger exposures may require surgical correction if economically practical. Affected chicks should be monitored carefully for omphalitis or yolk sac retention.

Omphalitis, or yolk sac infection, can be a multifactorial problem seen in chicks less than 1 week of age. Chicks that have been stressed by incorrect incubator/hatcher temperature and humidity or chicks that are weak or undersized at hatch are predisposed to bacterial infection. This infection usually arises from poor hygiene in the hatcher or brooder, although transovarial and transoviductal infections and shell contamination also can be involved. Many chicks that develop yolk sac retention may subsequently develop omphalitis. The incidence on 
Table 41.13 | Prevention of Omphalitis and

Yolk Sac Retention*

- Optimal incubation conditions

- Avoidance of early or inappropriate intervention during hatching (an exposed yolk sac has a much higher chance of becoming infected)

- Good hatcher hygiene

- Minimal handling after hatching

- Adequate umbilical care

- Adequate nutrition and exercise

*Black (personal communication)

many farms can be reduced by correcting incubator and hatcher problems and hygiene and applying an iodine ointment and bandage to the umbilicus of newly hatched chicks (Table 41.13). If chicks from a particular hen show a high incidence of omphalitis (without other chicks being affected), that hen needs to be investigated for the possibility of salpingitis.

Yolk sac retention, on the other hand, is a failure of the yolk sac to be resorbed in the absence of infection. This is primarily a management problem, with possible faults lying in incubator or hatcher problems, chick nutrition and exercise. Unless secondary omphalitis develops, clinical signs do not become evident until the chick is 2 to 6 weeks old and the autolyzing yolk begins to release possible toxic substances that are absorbed by the mesenteric blood vessels. These chicks fail to thrive and usually start to lose weight. The yolk sac often is palpable in the abdomen or is detectable via ultrasound.

As antibiotics do not penetrate the yolk sac, treatment for both omphalitis and yolk sac retention is the surgical excision of the yolk sac. Although a relatively simple procedure, the success rate for omphalectomy is not high due to pre-existing toxemia and immunosuppression.

\section{DISEASES OF THE MUSCULOSKELETAL SYSTEM}

"Wry neck" or torticollis is seen occasionally in all ratite species, with emus been the most frequently affected. The muscles and tendons of the neck cause abnormal positioning of the neck and head. Suggested etiologies include vitamin $\mathrm{E}$ and selenium deficiencies, teratogens, parental malnutrition, excessive handling and turning of the egg during incubation, and skeletal malformation. Splinting and dietary supplementation may improve many chicks. ${ }^{61}$

Tibiotarsal rotation (or angular limb deformity) is one of the most common and serious limb deformities seen in ratite chicks (Fig 41.21). The tibiotarsus rotates along its long axis, leading to an outward rotation of the limb (Fig 41.22 ). Mild cases may show only a "windmilling" gait when running, ie, the affected leg swings out rather than going straight ahead. Severe cases may be so badly rotated that the chick has great difficulty in walking and standing. Numerous causes have been suggested, indicating this problem's multifactorial nature. Possible etiologies may include diets excessively high in protein and/or energy, calcium/phosphorous imbalances, leg injuries, lack of exercise and heating of chick pen floors. Although no hereditary component has been conclusively identified, it appears that certain breeding pairs may produce offspring that have a high growth rate. These offspring, if fed a diet that can maintain or encourage this high growth rate, will develop a higher incidence of tibiotarsal rotation if they are exercised inadequately. Various surgical corrections have been described, but none has shown consistent long-term success. In kiwi chicks, "splayed legs", which occur when the brooder box substrate is too slippery to afford adequate traction, can readily be corrected with hobbles if identified in the early stages (Fig 41.23) (Jakob-Hoff, personal communication).

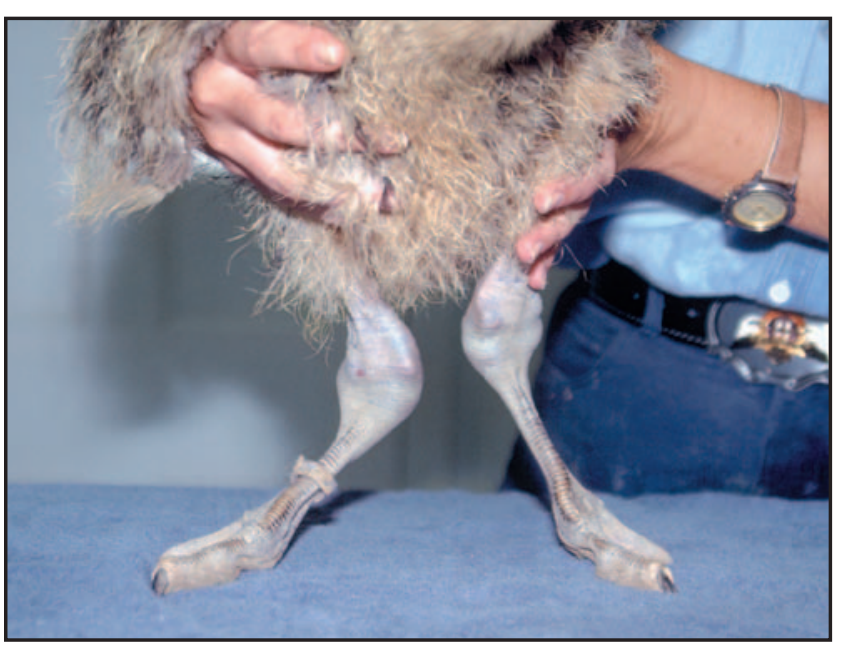

Fig 41.21 | Angular limb deformity in an ostrich chick raised on an oxalate-rich pasture, causing calcium deficiency.

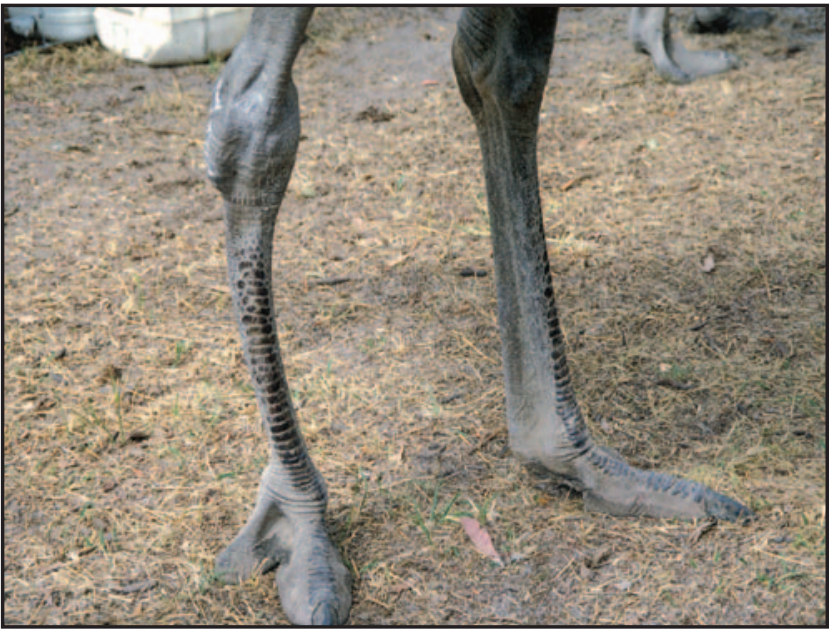

Fig 41.22 | Bowed tarsometatarsus, mature ostrich. This is usually the result of juvenile nutritional imbalance. 


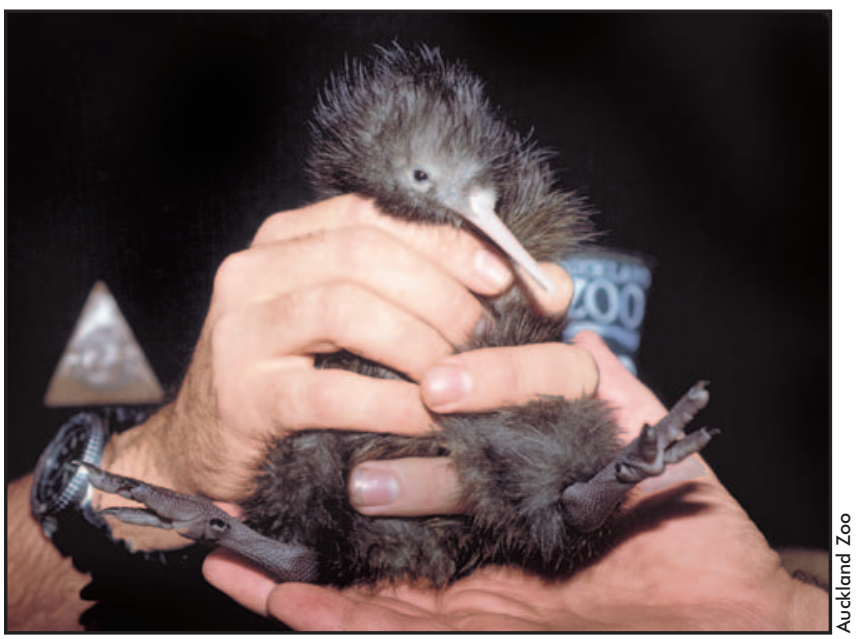

Fig 41.23 | Splay leg in a young kiwi chick. At this age, this condition is reversible through the application of hobbles and use of a non-slip substrate.

Rickets is the result of a deficiency of vitamin $\mathrm{D}_{3}$. This can be due to dietary imbalances, lack of sunlight or intestinal malabsorption. It results in enlargement of the joints and bowing of the femur, tibiotarsus and tarsometatarsus. ${ }^{61}$ Identifying and rectifying the inciting cause(s) may correct early cases. Other causes of bowed legs include nutritional calcium and phosphorous deficiencies or imbalances.

Rolled toes appear to occur in two distinct groups. The first group is chicks aged less than a week. Within a day or two of hatching, the toe begins to roll medially. Parental malnutrition and incubator errors have been implicated as causative factors. Corrective splinting or taping of the affected toe usually rectifies the roll within a few days. Chicks older than 4 to 5 weeks make up the second group. In these chicks, the rolled toe appears to be a variation of the angular limb deformity problem. Splinting may help with these chicks, but the prognosis is not as good unless dietary and exercise factors are corrected.

"Slipped tendon" refers to the lateral luxation of the flexor tendon of the gastrocnemius muscle out of its position on the caudal aspect of the hock joint. This can be mild, with immediate relocation of the tendon when the joint is straightened, or so severe that the tibiotarsus tears through the skin. Acute, mild cases may be successfully treated with bandaging and splinting. More severe cases, or cases where the luxation has been present for several hours, carry a poor prognosis. Surgical repair of the tendon retinaculum is required in these cases but is usually unsuccessful because of the weight-bearing stresses placed upon it.

\section{OSTRICH FADING SYNDROME (CHICK FADING SYNDROME)}

In the early 1990s, a syndrome in ostrich chicks less than 6 months old, characterized by weight loss, anemia and death, started to appear on ostrich farms throughout the world. Mortality rates vary from 20 to $80 \%$. Affected chicks progressively lose weight with wasting of the paralumbar muscles. Ascites often develops. Urate pasting around the vent is common as the chick weakens. A nonregenerative anemia is a frequent clinical finding.

Necropsy reveals a multitude of pathologies including rhinitis, pharyngitis, proventriculitis, enteritis, pneumonia, air sacculitis, hepatic abscessation and splenic atrophy. The most consistent histological finding is non-suppurative enteritis. ${ }^{13}$

The syndrome appears to have an infectious etiology, but numerous pathogens have been recovered from affected chicks: Cryptosporidia, gram-negative and gram-positive bacteria, fungi and yeast, and a range of viruses. No consistent pathogen has been identified. ${ }^{13,61}$

Given the wide range of findings and secondary pathogens isolated, it is generally presumed that a primary immunosuppressive agent is responsible for this syndrome. Work in Australia suggested that a retrovirus may be the causative agent, but this has yet to be confirmed. ${ }^{48}$

A major problem with this disease is the tendency of many farmers to categorize all chick mortalities as fading syndrome and decline further work-up. Clinicians must educate their clients against this tendency. The good news is that in recent years the incidence of this disease appears to have declined. Until the causative agent is identified, good biosecurity measures and treatment of secondary pathogens is the best that can be offered.

\section{Diseases Of Adult Ratites}

Mortality rates in ostriches decline rapidly with age. This appears to apply to all ratites, with few of the disease processes described for chicks applying to adult birds. Discussed below are some of the problems specific to adult birds, as well as some that can affect all age groups.

\section{DISEASES OF THE INTEGUMENT}

Skin lacerations are not uncommon in captive ratites, often occurring as injuries inflicted by collisions with wire fences. Although these respond well to basic treatment principles, it must be noted that scarring will significantly detract from the hide value in birds such as ostriches and emus. Fence construction and handling procedures must be done with the aim of minimizing such injuries. 
Bacterial and mycotic dermatitis and folliculitis do not appear to be common but can occur in excessively humid climates. Early recognition and treatment is essential to prevent or minimize damage to the hide.

An exudative dermatitis beginning around the external auditory meatus and progressing to involve the ventral and dorsal neck has been reported in captive kiwis whose normal vitamin/mineral supplement had been accidentally omitted from the diet. The condition responded dramatically to B-complex vitamin injections after 5 to 6 days. $^{10}$

Ectoparasites are common in ratites, with ticks, lice and mites being found on most species. While the primary clinical focus is on the damage caused to plumage and hide by lice such as the ostrich louse, Strutbioliperirus struthionis, and mites such as the quill mite, Gabucinia bicaudatus, other problems have been recorded. Tick paralysis, caused by an ixodid tick, Hyalomma truncatum, has been reported in South Africa, while an argasid tick, Argus persicus, can cause anemia in chicks and transmit aegyptianellosis from chickens to ostriches. ${ }^{21}$ The kiwi tick, Ixodes anatis, is suspected to be the vector of the Babesia spp. found in these birds. ${ }^{50}$ Regular spraying with $2 \%$ malathion or pyrethrins needs to be part of the husbandry of ratites.

\section{DISEASES OF THE DIGESTIVE SYSTEM}

Mycobacterial infections have been reported in ostriches, emus, cassowaries, rheas and kiwis. ${ }^{29,34,58}$ Affected birds typically show chronic weight loss accompanied by a marked leukocytosis. Necropsy shows multiple granulomas throughout the liver parenchyma and intestinal serosa, extending into the mucosa. Mycobacterium avium is usually identified either by culture or PCR. Although some reports ${ }^{58}$ contend that intradermal tuberculin testing is diagnostic, this is not confirmed by other reports. ${ }^{29}$ At this time, intradermal testing should be used to confirm the presence of the disease in a flock, rather than in individuals. Although the zoonotic potential of $M$. avium strains isolated from ratites has not been fully ascertained, treatment is not recommended and affected birds should be euthanized.

Sick or stressed ostriches of all ages may start to ingest foreign or indigestible material, which blocks (impacts) the proventriculus. As more material (high-fiber grasses, stones, dirt, sticks) is added, the proventriculus becomes distended, but no ingesta can move through. Affected birds become anorectic, weak and lose weight. They may regurgitate water after drinking. Fecal output diminishes or ceases altogether. The impacted proventriculus is usually palpable. Young birds can be treated by gastric lavage while suspended upside down. Older birds may require a proventriculotomy. ${ }^{34}$ Although some authors ${ }^{34}$ advocate against the use of mineral oil, it has been used successfully in sand and gravel impaction when combined with psyllium (Doneley, personal observation). Treatment is of value only if the original stressor has been removed, behavioral stress minimized and access to substrate and/or foreign material prevented. ${ }^{35}$

Eastern equine encephalitis (EEE) has been reported in emus in North America. Affected birds show depression, recumbency and hemorrhagic diarrhea, followed by death. Prevention revolves around minimizing exposure to biting insects and a vaccination protocol using a commercial equine inactivated EEE vaccine..$^{58}$

An acute central nervous system disease manifesting as extreme depression and convulsions has been observed in a number of captive North Island brown kiwi. Some fatalities have occurred while others have made a full recovery over a number of days to weeks with supportive care. Although response to antibiotics has suggested a bacterial etiology, not all affected birds responded similarly (Jakob-Hoff, personal communication).

\section{DISEASES OF THE RESPIRATORY SYSTEM}

The most common respiratory disease seen in ratites is air sacculitis associated with infection by Aspergillus spp. Unlike the "brooder pneumonia" seen in young chicks, this is a chronic disease with clinical signs often appearing within a few weeks of a stressful event such as transport. Affected birds lose weight and show an increased respiratory effort, seen as an increased respiratory rate (greater than 20 breaths per minute in a non-stressed bird), increased sternal lift and open-mouth breathing. Care must be taken to observe suspect birds before they are handled or heat stressed, as this can replicate the signs of respiratory distress. Some birds may cough. Auscultation over the paralumbar fossa may detect rasps, squeaks and friction rubs at peak inspiration and expiration. Leukocytosis is frequent, but serology is unreliable, as many birds appear to fail to seroconvert and will test falsely negative.

Aspergillosis is not contagious among birds, but it is not uncommon to find several birds infected from a common source. Treatment with antibiotics (for secondary infections), itraconazole orally and fumigation with enilconazole smoke bombs ${ }^{\mathrm{c}}$ appears to be effective in causing remission of clinical signs in many birds, although recurrence months or years later is common (Doneley, personal observations). ${ }^{43}$

Avian influenza, caused by a number of strains of the 